\title{
Numerical investigation on Hopf bifurcation and post-instability of tube bundles subjected to two-phase cross-flow and loose support
}

\author{
Jiang Lai ${ }^{\mathrm{a}, *, 1}$, Han Wu ${ }^{\mathrm{b}, 1}$, Lei Sun ${ }^{\mathrm{a}}$, Lixia Gao ${ }^{\mathrm{a}}$, Pengzhou $\mathrm{Li}^{\mathrm{a}}$ \\ a Nuclear Power Institute of China, Chengdu 610213, China \\ ${ }^{\mathrm{b}}$ Key Laboratory for Mechanics in Fluid Solid Coupling Systems, Chinese Academy of Sciences, Institute of Mechanics, Beijing 100190, China
}

\section{A R T I C L E I N F O}

\section{Article history:}

Received 8 February 2020

Received in revised form 26 February 2020

Accepted 7 March 2020

\section{Keywords:}

Nonlinear dynamics

Tube bundles

Two-phase flow

Bifurcation

Post-instability

\begin{abstract}
A B S T R A C T
This paper investigated the Hopf bifurcation and post-instability of tube bundles subjected to twophase cross-flow and loose support. A nonlinear dynamic model of a single tube in tube bundles considering the effects of two-phase flow force and impact force between the tube and tube support plate was established. Based on this model, a numerical simulation program was written to calculate the bifurcation diagrams, limit cycle motions, and quasi-periodic motions of the tube. The numerical results showed that a supercritical Hopf bifurcation of a single flexible tube in a tube array at the original stable equilibrium occurs at the Hopf bifurcation velocity within void fraction ranging from $0 \%$ to $80 \%$. And, the vibrations of the tube for the high void fraction condition are much more complex than those for the low void fraction which could be the reason for the early damage of the tube bundles in a steam generator.
\end{abstract}

(c) 2020 Elsevier Ltd. All rights reserved.

\section{Introduction}

Two-phase cross-flow-induced fluidelastic instability of the tube bundles in a steam generator is a key issue to the safety of a nuclear power plant. Since the 1960s, a great effort has been dedicated to this flow-induced instability of heat exchangers. The fluidelastic instability of a tube placed in an array subjected to two-phase cross-flow was studied by Joo and Dhir (1995). Their investigation illustrated that the onset of fluidelastic instability is associated with a rapid decrease of the effective spring constant. Chung and Chu (Chen et al., 1998) investigated the fluidelastic instability of two different arrays of straight tube bundles in two-phase cross-flow. The experimental results showed that the vibration characteristics of the rotated square array tube bundles in two-phase cross-flow were quite different from those of the normal square array tube bundle. Based on unsteady flow theory, Chen et al. (Chung and Chu, 2006) presented a mathematical model for fluid damping controlled instability of tubes in cross-flow, which can be used to predict structural instability

\footnotetext{
* Corresponding author.

E-mail address: laijiang1983@163.com (J. Lai).

1 These authors equally contributed to this work.
}

due to fluid damping. Chu et al. (2009) performed an experimental study to investigate the fluidelastic instability of U-tube bundles in air-water two-phase cross-flow, and the instability constant of the Connors equation was assessed with a simplified effective gap velocity. Mahon and Meskell (2009) studied the interaction between fluidelastic instability and acoustic resonance in a normal triangular tube array. They found that acoustic resonance could modify the time delay between the tube motion and flow field around the cylinder resulting in the drop in fluidelastic vibration amplitude. Ricciardi et al. (2011) presented an experimental study to analyze the vibration of a normal triangular tube array subject to air-water cross-flow. Comparing with the results obtained with a rotated triangular tube array, they found that the normal triangular configuration is more stable than the rotated triangular configuration. Zhao et al. (2014) used a fully coupled model for fluid dynamics and structure to analyze the flowinduced vibration of steam generator tubes. The dynamical behaviors of two tubes with in-line and parallel configuration were also studied. Bouzidi and Hassan (El and Bouzidi, 2015) developed a computational fluid dynamics model to calculate the fluid force inside the tube bundles. Then, considering the effect of the time lag of the fluid force on the threshold of fluidelastic instability was also investigated. Jiang et al. (2015) extended the streamtube model for fluidelastic instability in single-phase flow to two-phase flow by two kinds of two-phase 

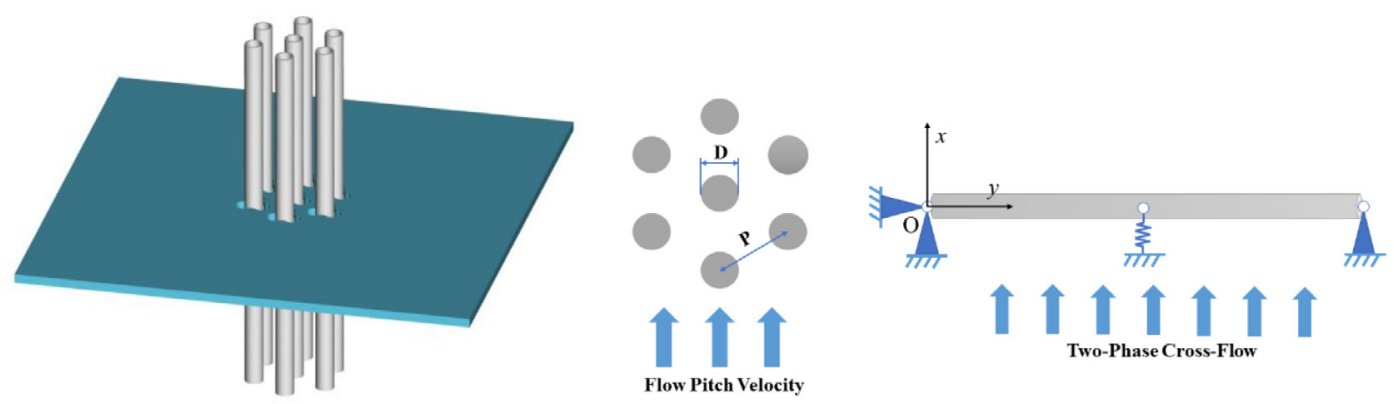

Fig. 1. Schematic diagram of a rotated triangular tube array subjected to two-phase cross-flow and loose support.

flow models which are beneficial for predicting the fluidelastic instability of tube bundles from existing stability maps and methods. Based on a bench-scale experimental setup, Liu et al. (2017) measured the fluid excitation forces acting on the tube bundles in a normal triangular tube array with water cross-flow, and presented a quasi-periodic mathematical model of fluid excitation forces acting on a circular cylinder. Jiang et al. (2017) presented a new set of upper bound of buffeting forces in two-phase flow

Table 1

Explanation and the values of the parameters.

\begin{tabular}{ll}
\hline Parameters & Values \\
\hline Elasticity modulus & $E=210 \mathrm{GPa}$ \\
Length of the tube & $L=0.312 \mathrm{~m}$ \\
Outer diameter of the tube & $D=0.01748 \mathrm{~m}$ \\
Pitch between tubes & $P=0.0259 \mathrm{~m}$ \\
Air density & $\rho_{G}=1.293 \mathrm{~kg} / \mathrm{m}^{3}$ \\
Water density & $\rho_{L}=1000 \mathrm{~kg} / \mathrm{m}^{3}$ \\
Void fraction reduction constant & $p=0.75$ \\
\hline
\end{tabular}

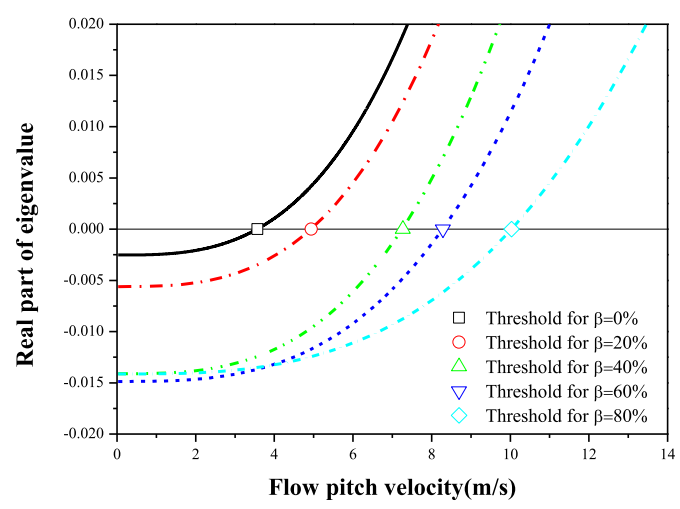

(a)

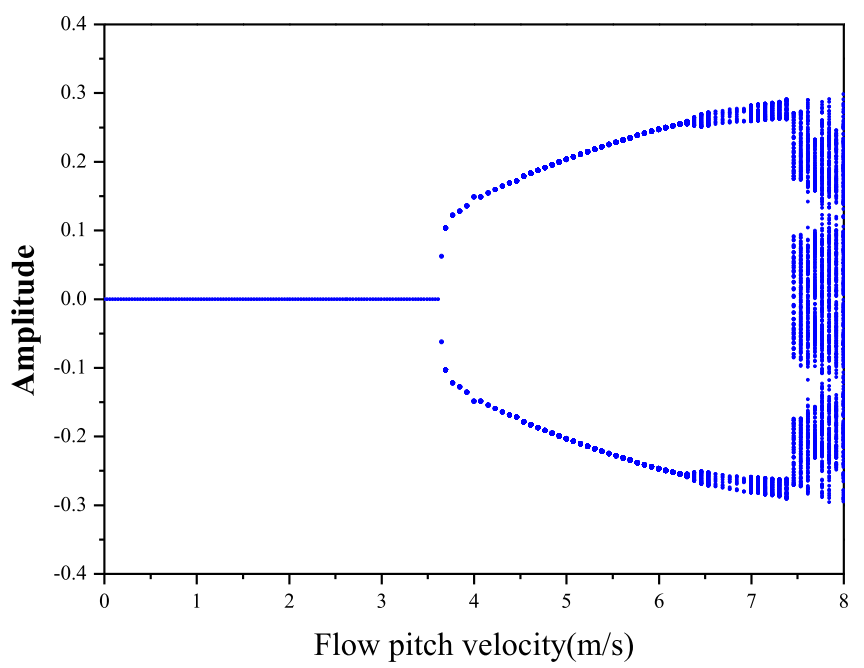

Fig. 3. The bifurcation diagram for the tube bundles with loose support in $0 \%$ void fraction.

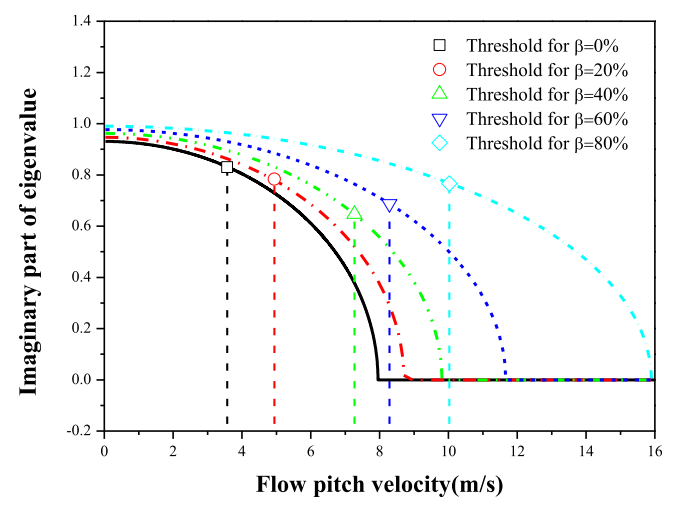

(b)

Fig. 2. The bifurcation diagram for the tube bundles with loose support in $0 \%$ void fraction.

Table 2

A comparison of the critical velocity of the fluidelastic instability for the five void fraction conditions.

\begin{tabular}{|c|c|c|c|c|c|}
\hline & $0 \%$ void fraction & $20 \%$ void fraction & $40 \%$ void fraction & $60 \%$ void fraction & $80 \%$ void fraction \\
\hline Hopf bifurcation velocity $(\mathrm{m} / \mathrm{s})$ & 3.57 & 4.94 & 7.27 & 8.29 & 10.03 \\
\hline Relative deviation & & $38.4 \%$ & $103.6 \%$ & $132.2 \%$ & $180.9 \%$ \\
\hline
\end{tabular}


by modifying the mixture velocity proposed by de Lange which has been compared with the one based on single-phase flow. Based on transient interaction between a single tube and adjacent flow streams of single-phase fluid, Shinde et al. (2018) presented a theoretical model of fluidelastic instability of a tube array. The critical velocity was also obtained as a function of mass ratio and damping parameter. To predict the unsteady fluid forces in a parallel triangular array subjected to two-phase flow, Sadek et al. (2018) presented a numerical model based on the RANS formulation with aid of Spalart-Allmaras turbulence model. And the stability was also investigated by studying the eigenvalues of the tube bundle system as a function of the flow pitch velocity. Ma et al. (2020) investigated the flow-induced vibration characteristics of three equally spaced cylinders using an inverse analysis method.

Recently, some studies on the nonlinear dynamics of the tube bundles subjected to single-phase flow and loose support have been reported. The experimental and numerical analyses on the problem of the nonlinear vibro-impact responses of loosely supported heat-exchanger tubes subjected to single-phase flow were performed by Piteau et al. (2012) Based on a five-mode discretization of the governing partial differential equation, Wang et al. (2012) analyzed the instability and nonlinear dynamics of planar motions of a cylinder array subject to single-phase crossflow considering the effect of the initial axial load. Their theoretical results indicated that the tube system may lose stability by increasing the initial axial load and flow velocity. Ni et al. (2015) investigated the nonlinear dynamics of a cantilevered pipe conveying fluid interacting with two support walls on both sides. Zhang et al. (2016) studied the effects of increasing and decreasing flow velocities on the fluidelastic instability of tube bundles in singlephase cross-flow. Their results showed that the nonlinear hysteresis phenomenon occurred in both tube bundle vibrations. Abdelbaki et al. (2018) presented a full nonlinear model for the dynamics of a cantilevered cylinder subjected to confined, inverted axial flow. Based on this model, the stability of the tube system was investigated by means of bifurcation diagrams, time histories, phase-plane, and power-spectral-density plots. The experimental and numerical studies were utilized to obtain the threshold of fluidelastic instability of a tube array subjected to two-phase crossflow by Lai et al. (2019). And the nonlinear dynamics of the tube array considering the effect of a clearance gap were further investigated.

As mentioned above, several experimental and numerical studies have been carried out to obtain the threshold of fluidelastic instability of a tube array subjected to two-phase flow, which is a typical eigenvalue problem without considering the effect of the constraint conditions of the tube bundles. However, in a steam generator, it is important to note that the tubes are always threaded through the tube support plates (TSP), or constrained by the anti-vibration bars (AVBs). Thus, the tube bundles system with TSP or AVBs processes nonlinear mechanism, and flowinduced vibration of the tubes may very complicated within the effect of two-phase flow. Despite the number of studies on flowinduced fluidelastic instability of the tube bundles, few have considered the influence of the two-phase cross-flow loads and loose supports. Therefore, it is still a problem deserving further investigations for the bifurcation and post-instability of the tube bundles with loose support in two-phase cross-flow. That is to say, what effects can the two-phase cross-flow have on the nonlinear dynamics of the tube bundles? Quantitative study has to be performed on this problem because the post-instability vibration of a single flexible tube in a tube array could be the reason for the early damage of the tube bundles in a steam generator.

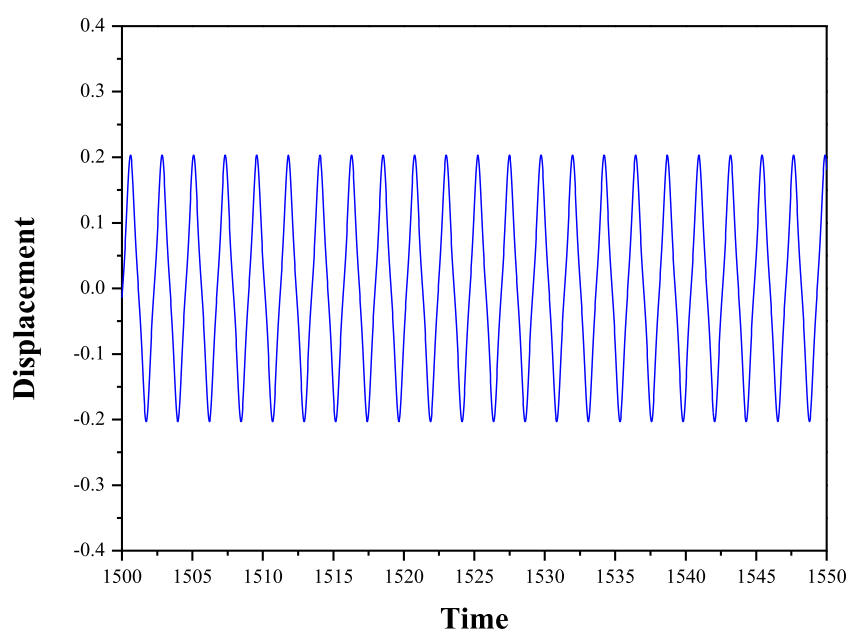

(a)

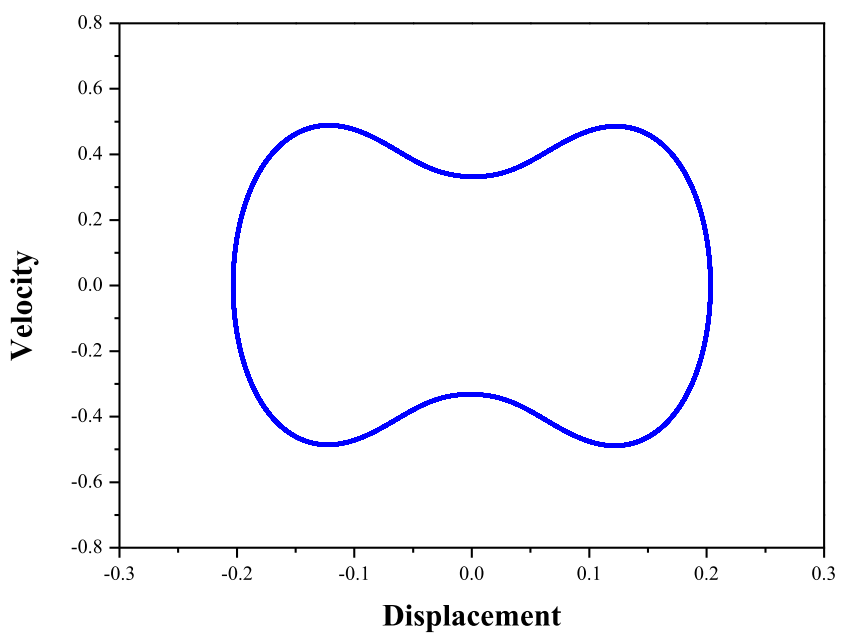

(b)

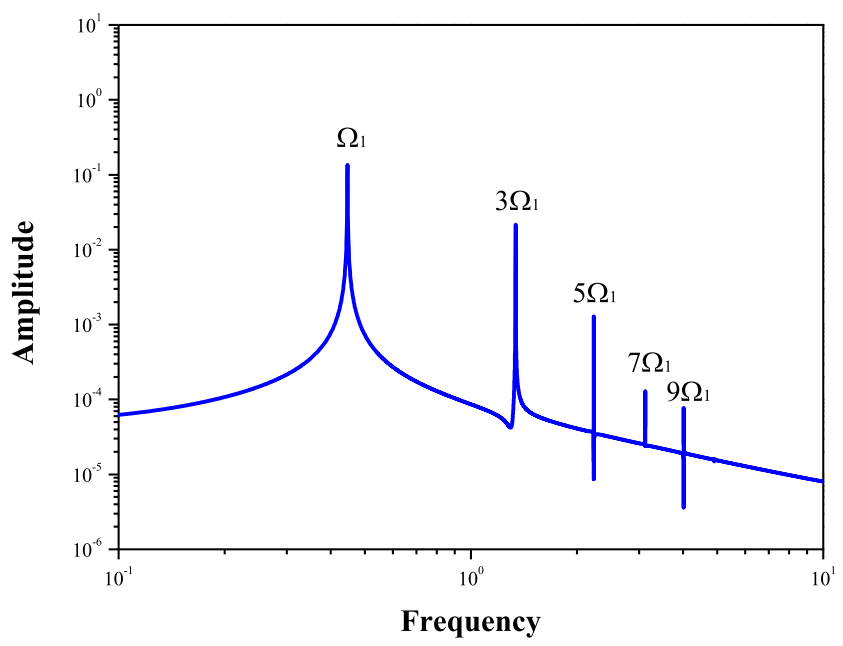

(c)

Fig. 4. Time history, phase-plane projection and spectrum of the tube in $0 \%$ void fraction when the flow pitch velocity is $4.998 \mathrm{~m} / \mathrm{s}$ : (a) displacement; (b) phase plane projection; (c) frequency spectrum. 
In this study, the eigenvalue problem of fluidelastic instability of a tube array in five void fraction conditions $(0 \%, 20 \%, 40 \%, 60 \%$, and $80 \%$ void fraction) was investigated firstly. Then, the effect of the loose support and void fraction of two-phase flow on the vibration characteristics of a single flexible tube in tube bundles was taken into account. We have used a path-following method to calculate the bifurcation diagrams for the tube bundles in the five void fraction conditions ( $0 \%, 20 \%, 40 \%, 60 \%$, and $80 \%$ void fraction). By analyzing the bifurcation characteristics, the nonlinear dynamics, we have determined the influence of loose support and twophase flow on the post-instability of the tube bundles.

\section{Theoretical model}

Fig. 1(a) shows a dynamics model of a rotate triangular tube array subjected to two-phase cross-flow and the tube support plate. In the event of one tube support plate failure, the tube bundles can be regarded as two-span tube clamped at both ends and loosely supported at the middle, as showed in Fig. 1(b). The equation of motion for a single flexible tube in a rotated triangular tube array subjected to two-phase cross-flow and loose support can be expressed as:

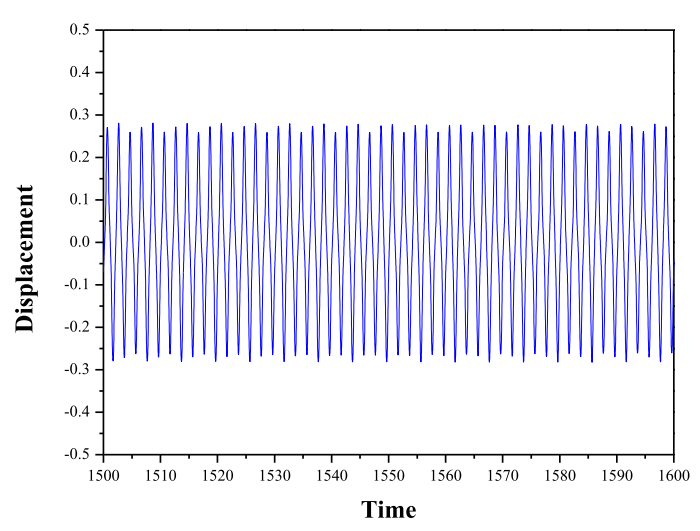

(a)

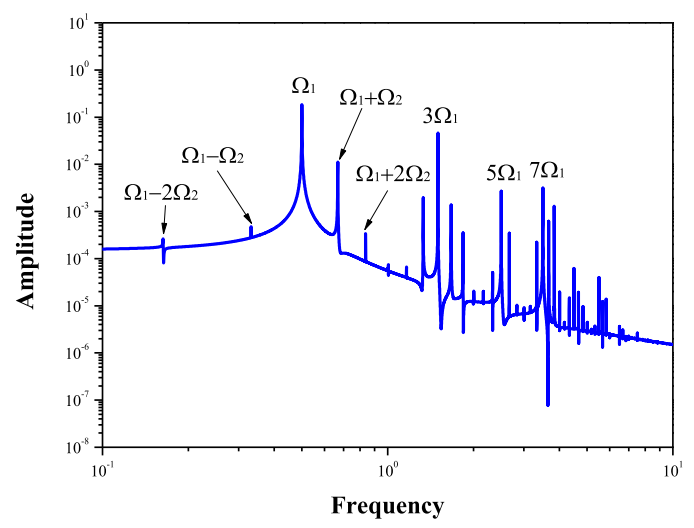

(c)
$E I \frac{\partial^{4} w}{\partial y^{4}}+c_{t} \frac{\partial w}{\partial t}+m_{t} \frac{\partial^{2} w}{\partial t^{2}}=\delta\left(y-y_{a}\right) F_{\text {stiffness }}(w)+F_{\text {unsteady }}(w, \dot{w}, \ddot{w})$.

where $E I$ is the rigidity modulus of the tube, $w$ is lateral displacement of the tube, $c_{t}$ and $m_{t}$ is the damping coefficient and the mass of the tube per unit length, $\delta\left(y-y_{a}\right)$ is the Dirac delta function, $F_{\text {stiffness }}(w)$ is the nonlinear force representing the effect of loose support, $F_{\text {unsteady }}(w, \dot{w}, \ddot{w})$ is the fluid force of the two-phase cross-flow, where dot and double dot indicate the velocity and acceleration of the tube, respectively.

According to our previous studies (Lai et al., 2019), it is known that the unsteady fluid force model can be used to predict the threshold of fluidelastic instability of a rotated triangular tube array in two-phase cross-flow. And, $F_{\text {unsteady }}(w, \dot{w}, \ddot{w})$ can be expressed as:

$F_{\text {unsteady }}(w, \dot{w}, \ddot{w})=m_{a} \frac{\partial^{2} w(y, t)}{\partial t^{2}}+c_{a} \frac{\partial w(y, t)}{\partial t}+k_{a} w(y, t)$

where, the added mass of two-phase flow, $m_{a}$, the damping, $c_{a}$, and the stiffness, $k_{a}$, can be written as:

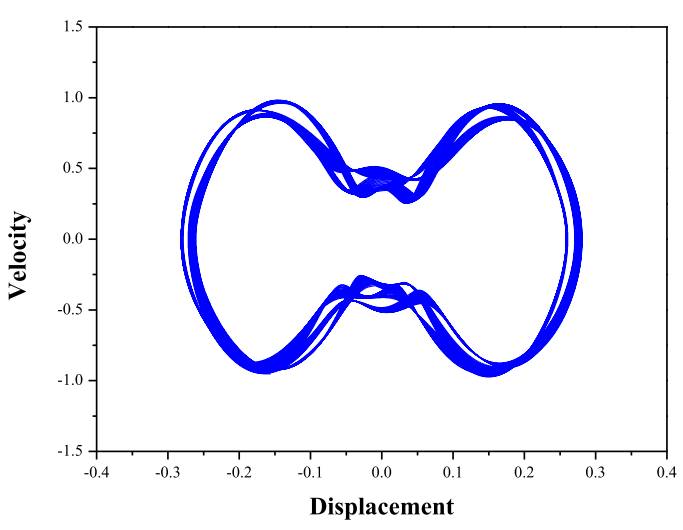

(b)

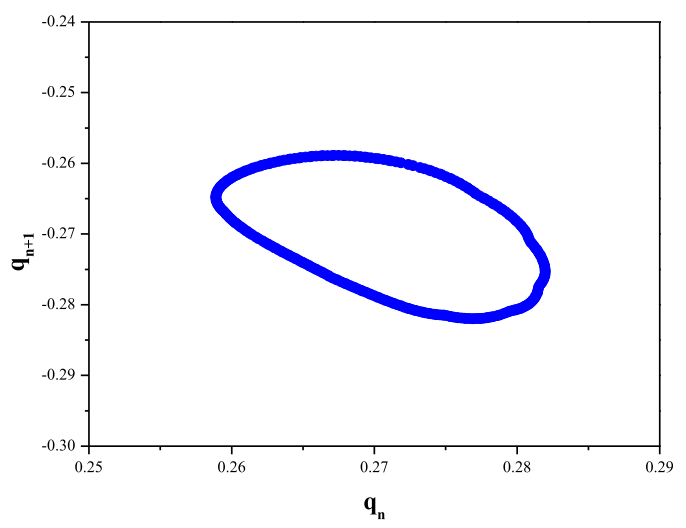

(d)

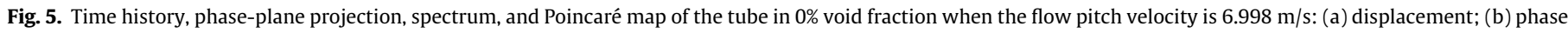
plane projection; (c) frequency spectrum; (d) Poincaré map. 


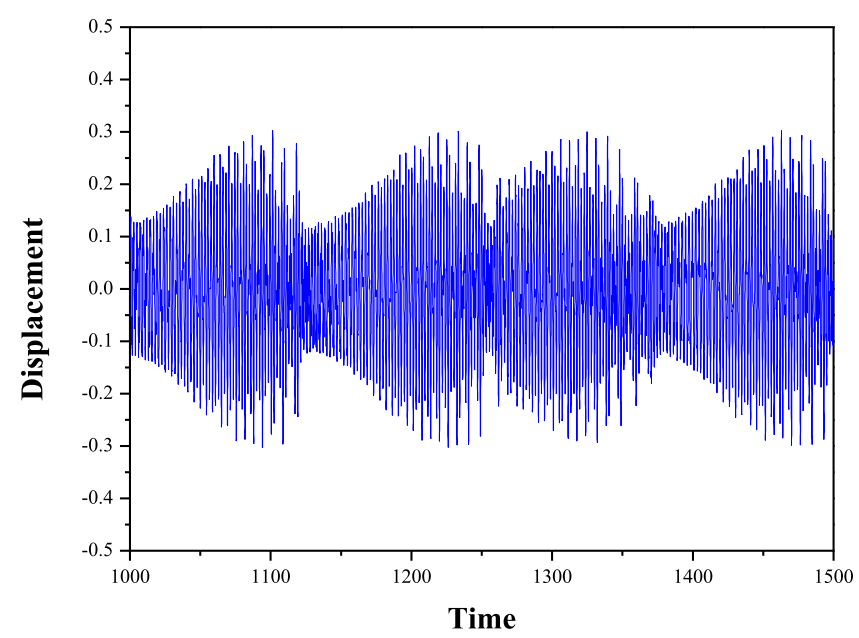

(a)

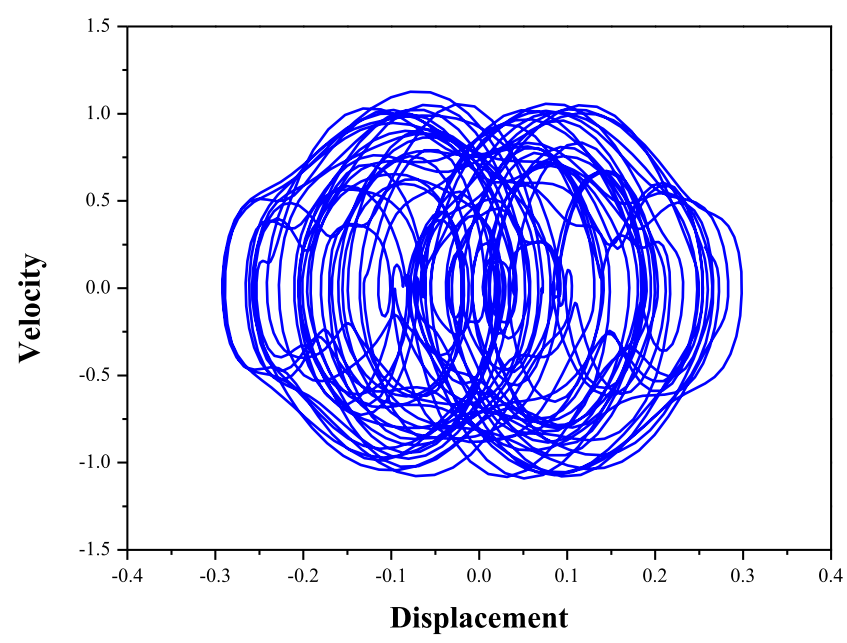

(b)

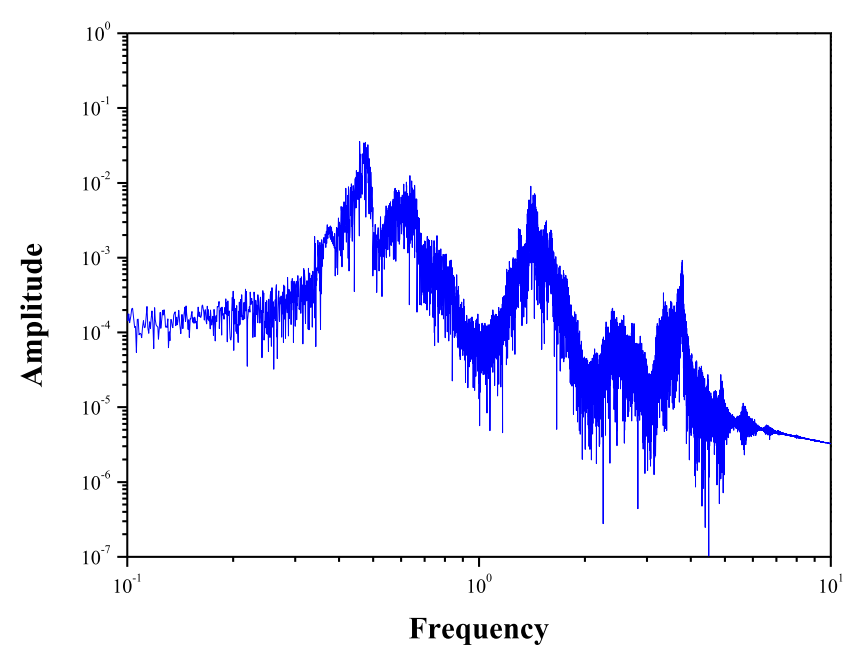

(c)

Fig. 6. Time history, phase-plane projection and spectrum of the tube in $0 \%$ void fraction when the flow pitch velocity is $7.998 \mathrm{~m} / \mathrm{s}$ : (a) displacement; (b) phase plane projection; (c) frequency spectrum.

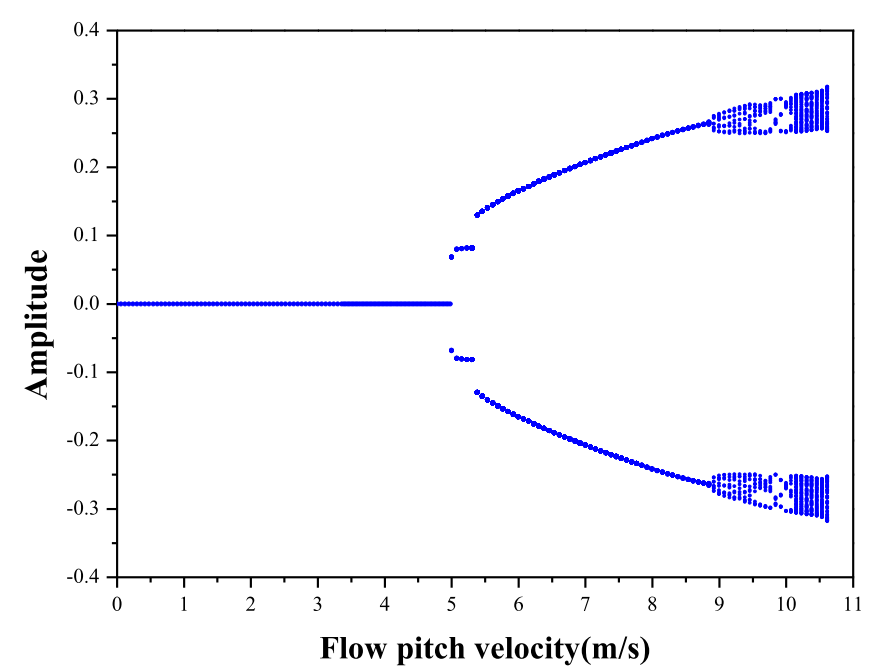

Fig. 7. The bifurcation diagram for the tube bundles with loose support in $20 \%$ void fraction.

$$
\begin{aligned}
& m_{a}=\frac{\pi}{4}\left[\rho_{G} p \beta+\rho_{L}(1-p \beta)\right] D^{2}\left[\frac{\left(D_{e} / D\right)^{2}+1}{\left(D_{e} / D\right)^{2}-1}\right] \\
& c_{a}=\frac{\rho U_{\infty}^{2} C_{F} \cos \Phi_{F}}{2} \\
& k_{a}=\frac{\rho U_{\infty}^{2} C_{F} \sin \Phi_{F}}{2 \omega}
\end{aligned}
$$

where $\rho_{G}$ is the air density, $\rho_{L}$ is the water density, $\rho$ is the twophase flow density, $\beta$ is the void fraction of two-phase flow, $D, D_{e}$ is the tube diameter and pitch between tube bundles, respectively, $U_{\infty}$ is the flow pitch velocity, $\omega$ is the angular frequency of the tube, $C_{F}, \Phi_{F}$ is the unsteady fluid force coefficient magnitude and phase, respectively.Introducing the following the non-dimensional quantities:

$$
\begin{aligned}
& \eta=\frac{w}{D}, \quad \xi=\frac{y}{L}, \tau=\lambda_{1}^{2} \sqrt{\frac{E I}{m_{t} L^{4}}} t=\Omega t, \varsigma=\frac{c_{t}}{\Omega m_{t}}, m^{*}=\frac{m_{t}}{\rho D^{2}}, U^{*}=\frac{2 \pi U_{\infty}}{D \Omega}, \\
& \alpha=\frac{1}{1+4 m^{*} /\left(\pi C_{m a}\right)}, \quad \omega^{*}=\frac{\omega}{\Omega}
\end{aligned}
$$

where $L$ is the tube length, $\lambda_{1}$ is the dimensionless eigenvalue of the first-order mode for a simply supported beam.

By substituting these dimensionless quantities into Eq. (1), the partial differential equation of the motion of the tube bundles considering the effects of two-phase cross-flow and loose support can be rewritten as:

$$
\begin{gathered}
\frac{1}{1-\alpha} \frac{\partial^{2} \eta}{\partial \tau^{2}}(\xi, \tau)+\left[\zeta-\frac{U^{* 2} C_{F} \sin \Phi_{F}}{8 \pi^{2} m^{*} \omega^{*}}\right] \frac{\partial \eta}{\partial \tau}(\xi, \tau)+\frac{1}{\lambda_{1}^{4}} \frac{\partial^{4} \eta}{\partial \zeta^{4}}(\xi, \tau) \\
-\left[\frac{U^{* 2} C_{F} \cos \Phi_{F}}{8 \pi^{2} m^{*}}-\frac{\alpha \omega^{* 2}}{1-\alpha}\right] \eta(\xi, \tau)+\delta\left(\xi-\xi_{b}\right) f^{*}(\eta)=0
\end{gathered}
$$

According to the Galerkin method, it is reasonable to suppose that:

$\eta(\xi, \tau)=\sum_{i=1}^{N} \varphi_{i}(\xi) q_{i}(\tau)$

where $\varphi_{i}(\xi)$ is the modal shape of the simple supported tube. It is known that the dynamic response of a tube is dominated by the first low modes. Thus, the first five order modes were chosen in present study.

Using the Galerkin expansion and modal truncation techniques, a set of ordinary differential equations can be deduced from the partial differential equation, as follows: 


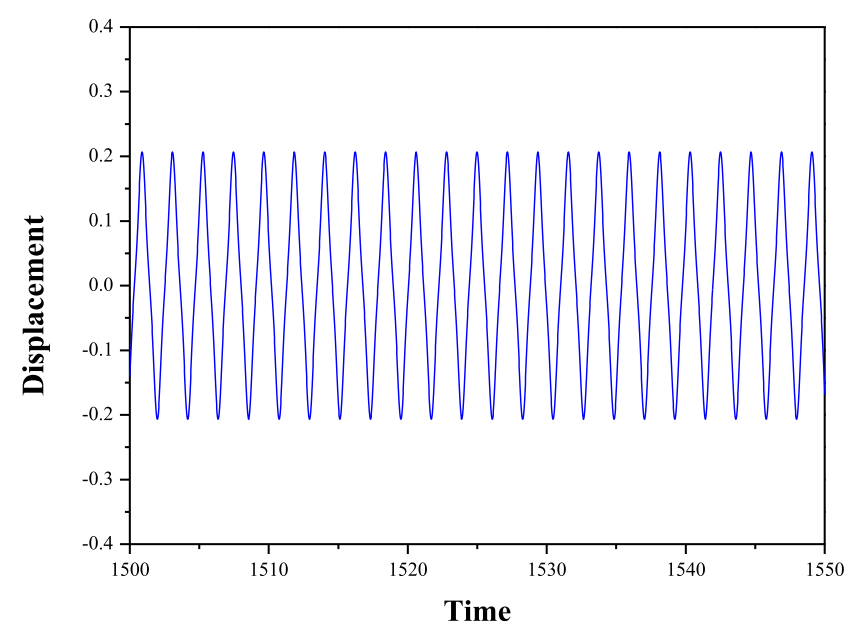

(a)

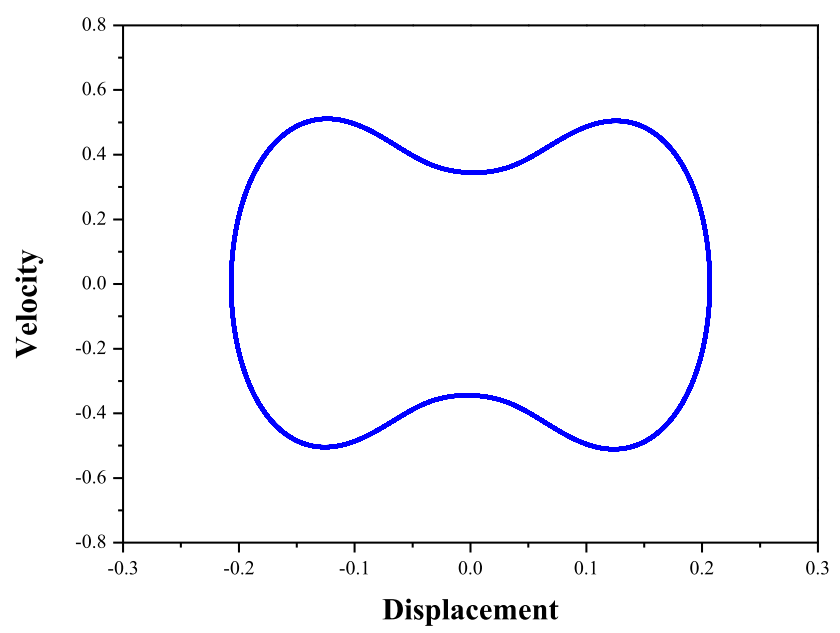

(b)

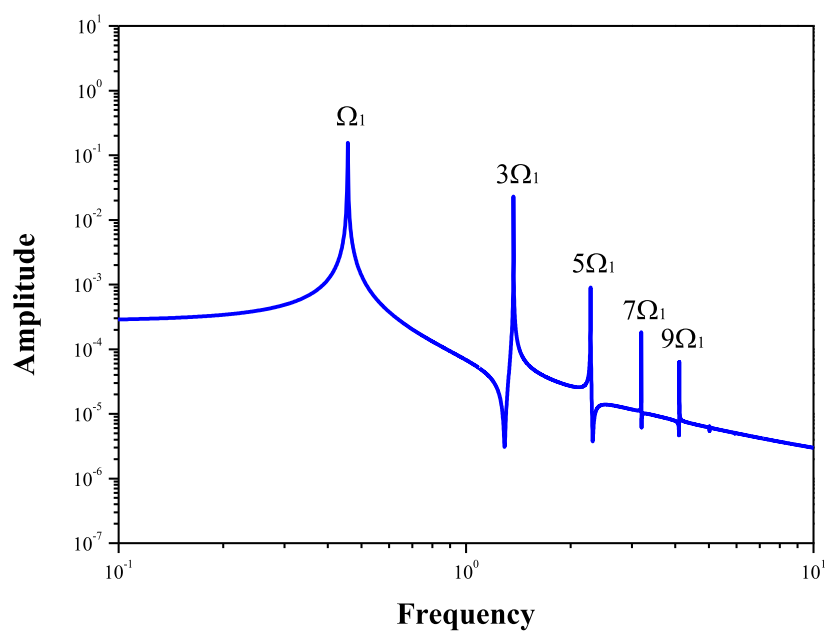

(c)

Fig. 8. Time history, phase-plane projection and spectrum of the tube in $20 \%$ void fraction when the flow pitch velocity is $6.998 \mathrm{~m} / \mathrm{s}$ : (a) displacement; (b) phase plane projection; (c) frequency spectrum.

$$
\begin{aligned}
& \frac{\ddot{q}_{i}}{1-\alpha}+\left(\zeta-\frac{U^{* 2} C_{F} \sin \Phi_{F}}{8 \pi^{2} m^{*} \omega^{*}}\right) \dot{q}_{i}+\left(\frac{\lambda_{i}^{4}}{\lambda_{1}^{4}}-\frac{U^{* 2} C_{F} \cos \Phi_{F}}{8 \pi^{2} m^{*}}+\frac{\alpha \omega^{* 2}}{1-\alpha}\right) q_{i}+f^{*}\left(\eta_{a}\right) \varphi_{i}\left(\xi_{a}\right)=0 \\
& (i=1,2,3,4,5)
\end{aligned}
$$

where $\eta_{a}$ is the displacement at $\xi=\xi_{a}$.

Notice that the coupling term is the impact force between the tube and tube support plate $f^{*}\left(\eta_{a}\right)$ due to the constraint at the loose support. In this study, the mathematical model presented by Paidoussis et al. (Paidoussis et al., 1989) (Paidoussis et al., 1991) was used to represent properly the restraining force of loose support, which can be written as:

$f^{*}\left(\eta_{a}\right)=\kappa \eta_{a}^{3}$

where $\kappa$ is the nondimensional stiffness of the cubic spring. In this paper, $\kappa$ is chosen to be 1000 in accordance with reference (Paidoussis and Li, 1992).

\section{Eigenvalue analysis}

To investigate the fluidelastic instability of the tube bundles considering the two-phase flow load, we analyze the eigenvalue problem for the linearized system of the tube bundles. The linearized equations at the equilibrium position can be written as:

$\mathbf{M}(\alpha) \ddot{\mathbf{Q}}+\mathbf{C}\left(\zeta, U^{*}, m^{*}, \omega^{*}, C_{F}, \Phi_{F}\right) \dot{\mathbf{Q}}+\mathbf{K}\left(U^{*}, m^{*}, \omega^{*}, C_{F}, \Phi_{F}, \alpha, \lambda_{1}\right) \mathbf{Q}=\mathbf{0}$

Eq. (11) is the reduced-order equation, where $q=(0,0)$ represents the equilibrium position.

$\dot{\mathbf{Q}}=[A] \mathbf{Q}, \quad[A]=\left.\left[\frac{\partial f_{i}}{\partial q_{j}}\right]\right|_{q=(0,0)}, \quad i, j=1,2, \cdots, 5$

According to the Lyapunov's indirect method, the stability of the nonlinear system described by Eq. (7) in the neighborhood of the tube equilibrium point can be determined by the eigenvalues of Jacobian matrix $A$. If all the real parts of the eigenvalues of the Jacobian matrix $A$ are negative, the tube bundles are stable. If a real part of the eigenvalues is zero, the tube system is at the critical state, and the corresponding flow pitch velocity is the threshold of fluidelastic instability.

In addition, it is obvious that, without the impact force between the tube and tube support plate, the mass matrix $\mathbf{M}(\alpha)$, damping matrix $\mathbf{C}\left(\xi, U^{*}, m^{*}, \omega^{*}, C_{F}, \Phi_{F}\right)$, and stiffness matrix $\mathbf{K}\left(U^{*}, m^{*}, \omega^{*}\right.$, $\left.C_{F}, \Phi_{F}, \alpha, \lambda_{1}\right)$ is diagonal matrix, respectively, which can be expressed as:

$$
\begin{aligned}
& \mathbf{M}=\operatorname{diag}\left[\frac{1}{1-\alpha}\right] \\
& \mathbf{C}=\operatorname{diag}\left[\zeta-\frac{U^{* 2} C_{F} \sin \Phi_{F}}{8 \pi^{2} m^{*} \omega^{*}}\right] \\
& \mathbf{K}=\operatorname{diag}\left[\frac{\lambda_{i}^{4}}{\lambda_{1}^{4}}-\frac{U^{* 2} C_{F} \cos \Phi_{F}}{8 \pi^{2} m^{*}}+\frac{\alpha \omega^{* 2}}{1-\alpha}\right]
\end{aligned}
$$

Based on our previous study (Liu et al., 2019), it is known that the first-order mode of the tube may become unstable firstly. Thus, the eigenvalues of the first-order mode corresponding to fluidelastic instability of a tube in the rotated triangular tube array were calculated for five different cases $(\beta=0 \%, 20 \%, 40 \%$, $60 \%$, and $80 \%$ ). The values of the parameter were illustrated in Table 1.

The results are presented in Fig. 2. The flow pitch velocity corresponding to the zero-real part of the eigenvalue is the 


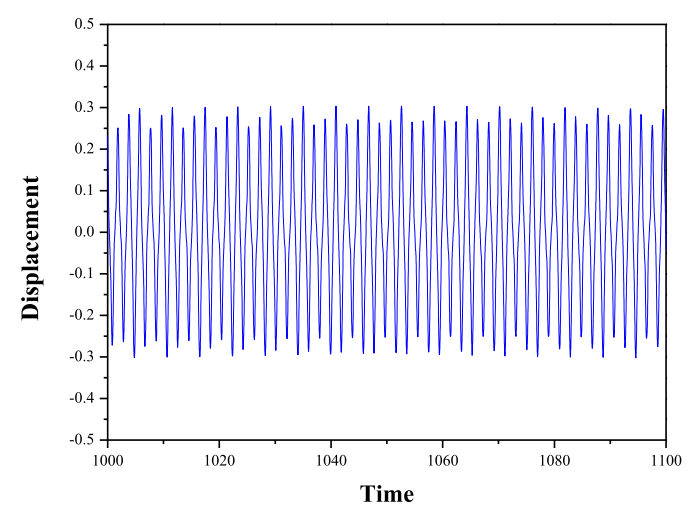

(a)

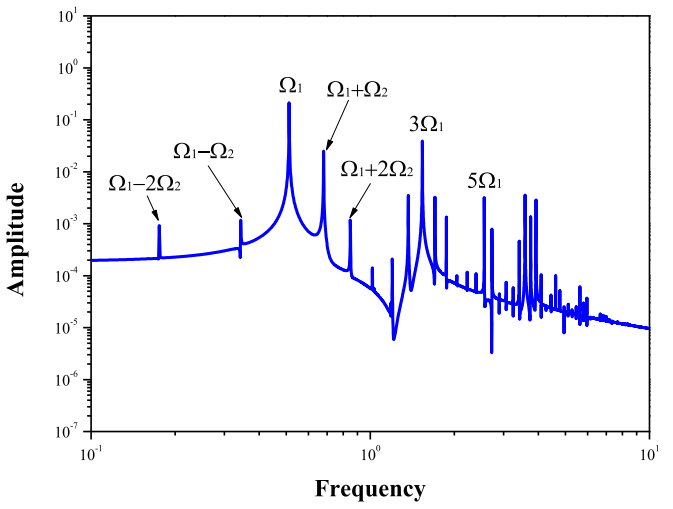

(c)

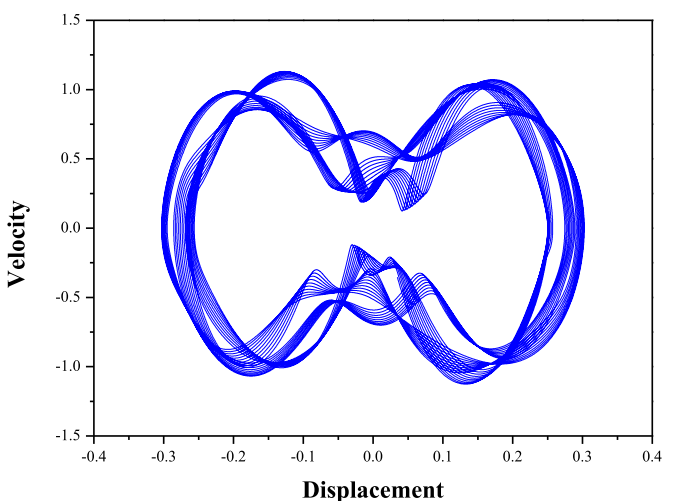

(b)

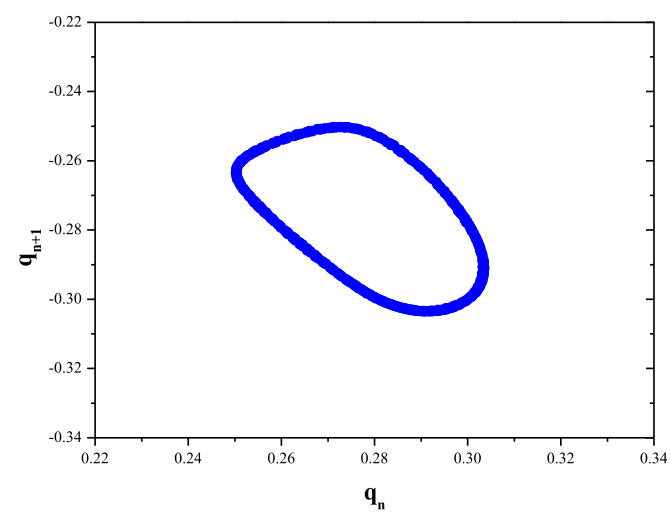

(d)

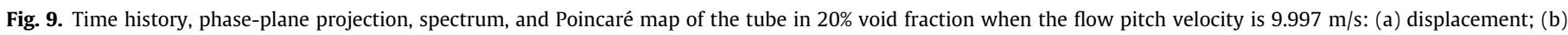
phase plane projection; (c) frequency spectrum; (d) Poincaré map.

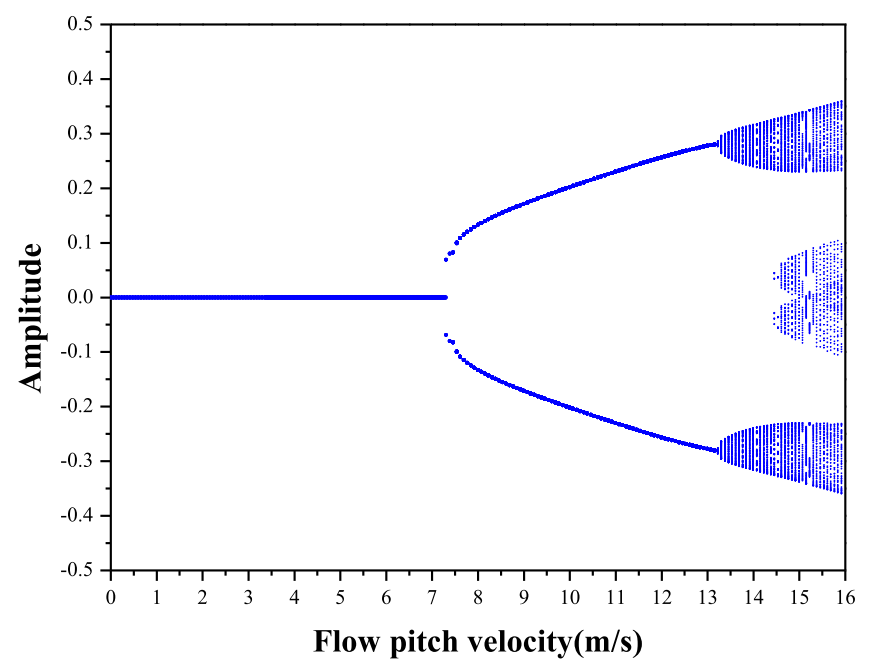

Fig. 10. The bifurcation diagram for the tube bundles with loose support in $40 \%$ void fraction. critical velocity. It is clearly seen that the threshold of fluidelastic instability of the tube increases monotonously as the void fraction of the two-phase flow increases. For the example considered in this study, the critical velocity of fluidelastic instability in two-phase flow for $20 \%, 40 \%, 60 \%$, and $80 \%$ void fraction is $4.94 \mathrm{~m} / \mathrm{s}, 7.27 \mathrm{~m} /$ $\mathrm{s}, \quad 8.29 \mathrm{~m} / \mathrm{s}, 10.03 \mathrm{~m} / \mathrm{s}$, which is $38.4 \%, 103.6 \%, 132.2 \%$, and $180.9 \%$ more than the critical velocity in single-phase flow. A direct comparison of the critical velocity of the fluidelastic instability for the five void fraction conditions was illustrated in Table 2. It is obvious that the real part of the eigenvalue increases with the increasing void fraction of two-phase flow, which means that in the high void fraction condition, the tube bundles are more stable. Furthermore, it also can be seen that the void fraction of two-phase flow has a significant effect on the damped frequency of the tube, as showed in Fig. 2(b). With increasing of the flow pitch velocity, once the flow pitch velocity exceeds the threshold of fluidelastic instability, the tube bundles could impact with the tube support plates, the tube modes become coupled. The periodic motion may occur, and the vibration characteristics of the tube bundles may become complicated. 


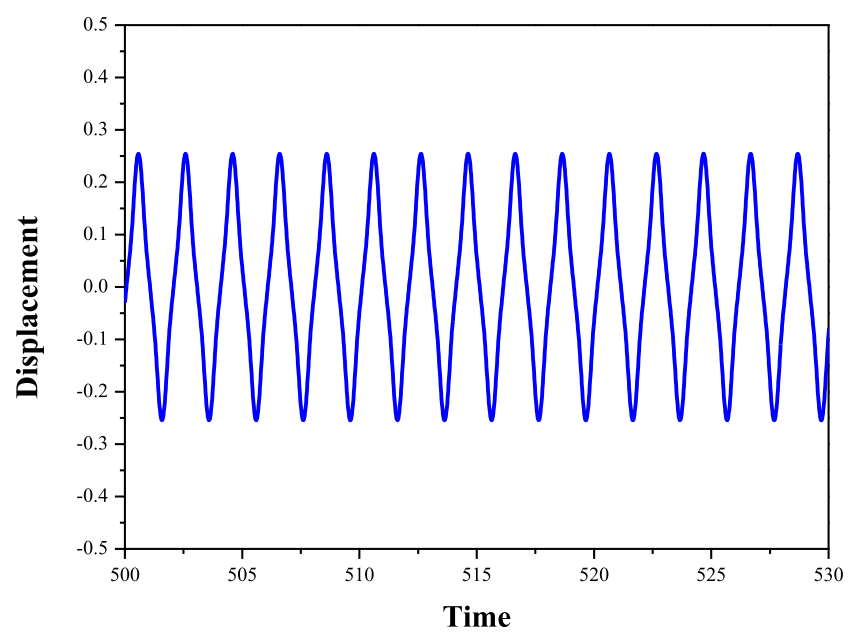

(a)

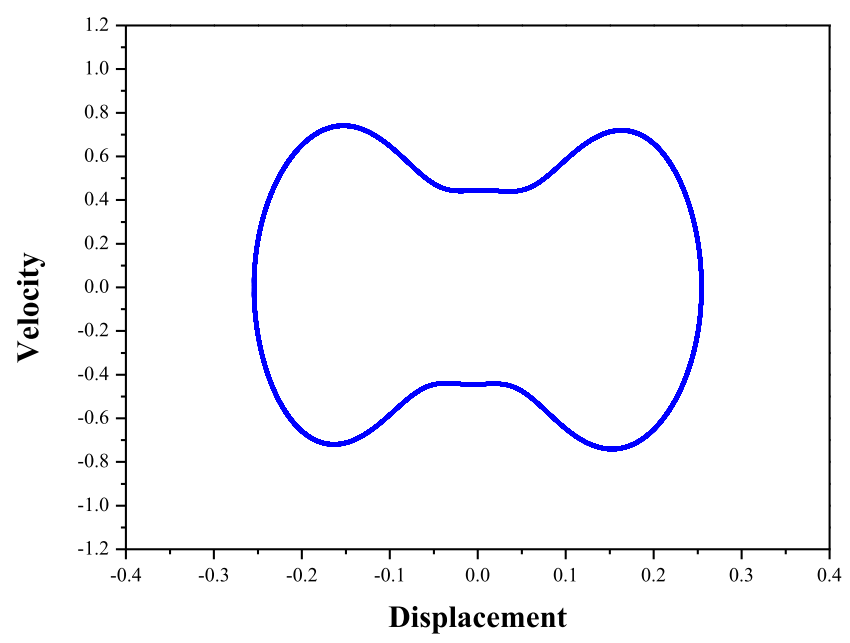

(b)

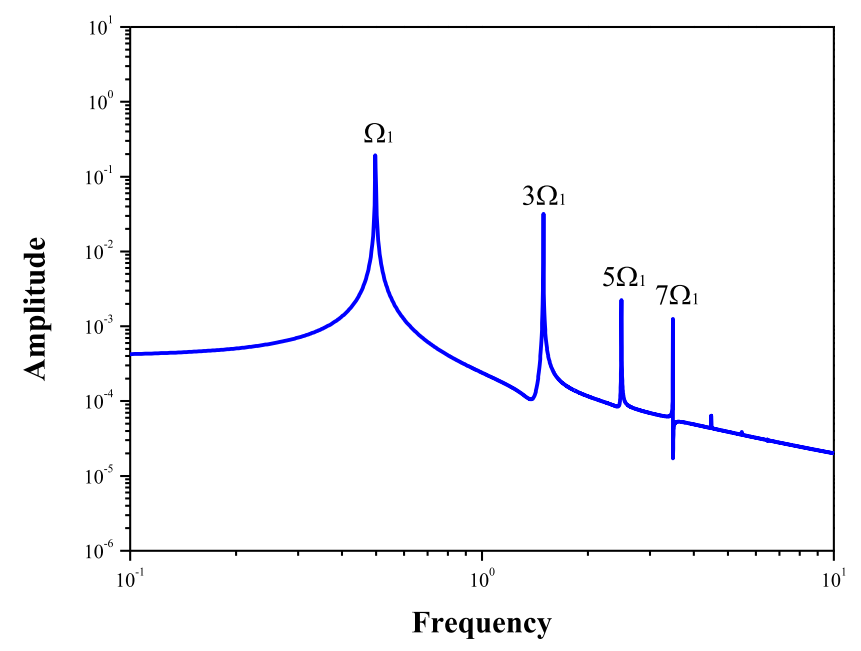

(c)

Fig. 11. Time history, phase-plane projection and spectrum of the tube in $40 \%$ void fraction when the flow pitch velocity is $11.919 \mathrm{~m} / \mathrm{s}$ : (a) displacement; (b) phase plane projection; (c) frequency spectrum.

\section{Nonlinear dynamic analysis}

On the basis of our dynamic model described above, the dynamic response of the tube was calculated by using a fourthorder Runge-Kutta integration algorithm, with a step size of 0.02 . The bifurcation diagrams of the tube subjected to two-phase cross-flow and loose support were constructed by plotting the peak-to-peak value of the vibration displacement of the tube in the transverse direction, in this case, the flow pitch velocity being the bifurcation parameter. In the following discussion, we focus on five void fraction conditions: $0 \%, 20 \%, 40 \%, 60 \%$, and $80 \%$ void fractions.

Fig. 3 shows the bifurcation diagram of the tube in $0 \%$ void fraction. The fluidelastic instability of the tube occurs at $\mathrm{U}_{\mathrm{p}}=3.614 \mathrm{~m} / \mathrm{s}$, resulting of a Hopf bifurcation at the original equilibrium position, which is equal to the numerical result obtained by eigenvalue analysis in the last section. It is obvious to see that once the flow pitch velocity exceeds the threshold of fluidelastic instability, the limit cycle motion of the tube occurs. The flow pitch velocity at the critical state where the limit cycle motion occurs is called the Hopf bifurcation velocity. It is important to note that the instability of the tube in the transverse direction is the supercritical Hopf type. Hence, the tube oscillation may decay to the original equilibrium position, when the flow pitch velocity of two-phase cross-flow is less than the threshold of fluidelastic instability for all initial conditions. On the other hand, when the flow pitch velocity is larger than the Hopf bifurcation velocity, as the flow pitch velocity is increased, the vibration amplitude of the limit cycle motion quickly grows. With increasing the flow pitch velocity continuously, the effect of the impact force between the tube and loose support could become apparent. Over most of the flow pitch velocity concerned range in present study, bifurcation results in transition between periodic solutions. However, within the flow pitch velocity ranging from $6.306 \mathrm{~m} / \mathrm{s}$ to $7.382 \mathrm{~m} / \mathrm{s}$, a significant quasiperiodic regime can be manifested. When the flow pitch velocity is larger than $7.459 \mathrm{~m} / \mathrm{s}$, another significant non-periodic regime can also be manifested. We now turn to a closer investigation of the resulting responses of the tube for the three typical regimes, the periodic motion, quasi-periodic motion, and non-periodic motion, respectively.

Fig. 4 shows the time history, phase-plane projection, and frequency spectrum of the tube in $0 \%$ void fraction at flow pitch velocity is $4.998 \mathrm{~m} / \mathrm{s}$, respectively. It can be clearly seen that the time series are shown in Fig. 4(a) has a flat envelope and no constant offset, and the signal is not sinusoidal in form. We can expect this signal to contain harmonics of the basic frequency. The velocity and displacement of the tube in $0 \%$ void fraction at $U_{p}=4.998 \mathrm{~m} / \mathrm{s}$ were used to define the state space, and a typical limit cycle motion can be seen in Fig. 4(b). The associated frequency spectrum of the tube in $0 \%$ void fraction was shown in Fig. 4(c). Unlike a linear tube bundles system, with the nonlinear effect of the loose support, higher frequency vibrations of the tube occur at multiples of the fundamental frequency, the odd frequencies appear in addition to the fundamental frequency. Fig. 5 shows the time history, phase-plane projection, frequency spectrum, and Poincaré map of the tube in $0 \%$ void fraction at $U_{p}=6.998 \mathrm{~m} / \mathrm{s}$, respectively. Fig. 5(a) shows an atypical periodic motion, and the phase-plane projection is more complex. It is important to note that a typical quasiperiodic motion occurs. This quasiperiodic solution is a dynamic solution characterized by two incommensurate frequencies, $\Omega_{1}$ and $\Omega_{2}$, as illustrated in Fig. 5(c). Thus, this quasiperiodic solution can be called as a two-periodic quasiperiodic solution. A 


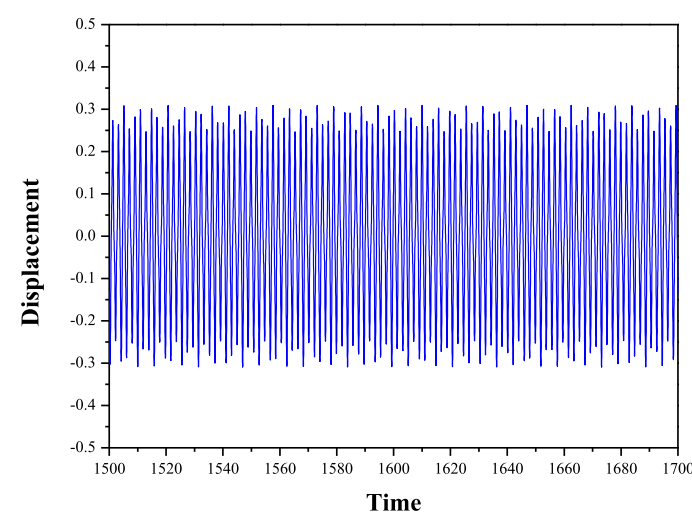

(a)

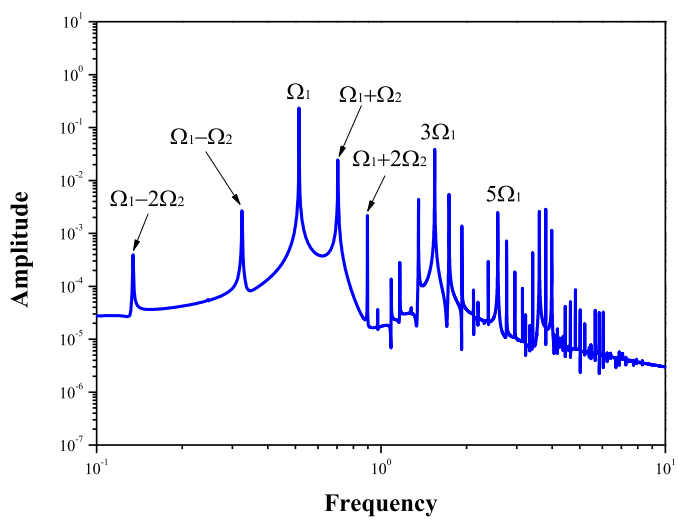

(c)

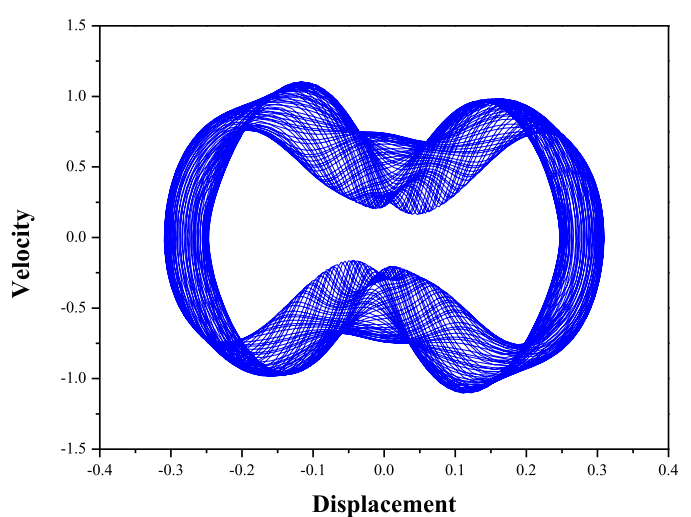

(b)

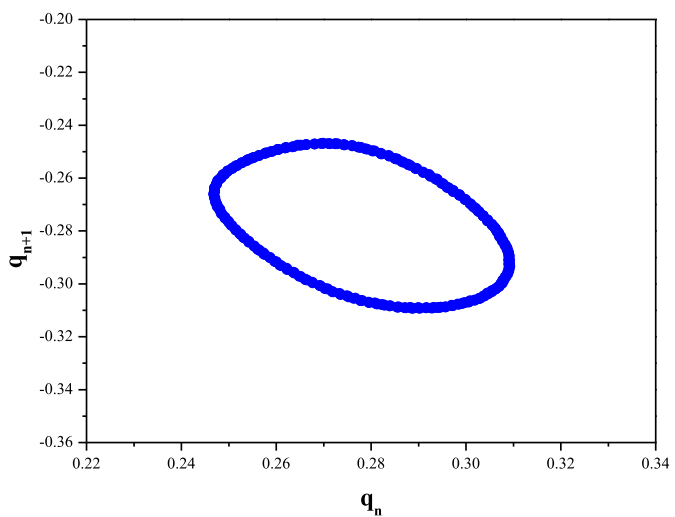

(d)

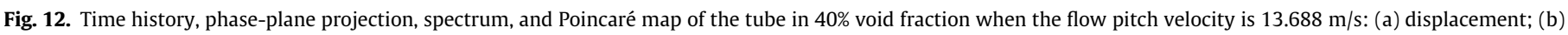
phase plane projection; (c) frequency spectrum; (d) Poincaré map.

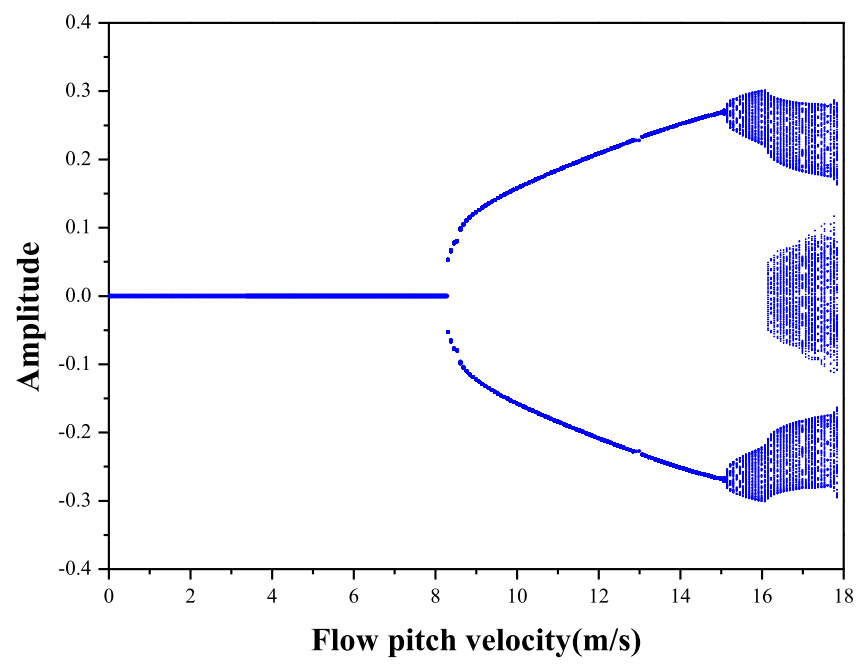

Fig. 13. The bifurcation diagram for the tube bundles with loose support in $60 \%$ void fraction. two-dimensional system was utilized to construct a Poincaré section, and the discrete points fall on a closed curve shown in Fig. 5 (d). Fig. 6 shows the time history, phase-plane projection, and frequency spectrum of the tube in $0 \%$ void fraction at $U_{p}=7.998 \mathrm{~m} /$ $\mathrm{s}$, respectively. At $\mathrm{U}_{\mathrm{p}}=7.998 \mathrm{~m} / \mathrm{s}$, the vibration of the tube becomes much more complex and appears non-periodic motion. For this complex motion, although the significant periodic component exists, the harmonic, sub-harmonic and multi-harmonic response of the tube can also be observed in Fig. 6(a) and (c), which indicates that the flow pitch velocity has a great effect on the high-frequency vibration of the tube.

Fig. 7 displays the bifurcation diagram of the tube bundles in $20 \%$ void fraction, and the bifurcation parameter is the flow pitch velocity, as previously. It can be clearly seen that the Hopf bifurcation of the tube at the original equilibrium position occurs at $\mathrm{U}_{\mathrm{p}}=4.980 \mathrm{~m} / \mathrm{s}$. In a small range of flow pitch velocity above $U_{p}=5.306 \mathrm{~m} / \mathrm{s}$, as the flow pitch velocity is increased, the vibration amplitude of the limit cycle motion grows, but the changes have been small. Continue to increase the flow pitch velocity, it is interesting to note that a jump occurs from the stable branch 
to another stable branch, and the vibration amplitude of the periodic motion quickly grows. At $\mathrm{U}_{\mathrm{p}}=8.843 \mathrm{~m} / \mathrm{s}$, a quasi-periodic motion occurs. In the concerned flow pitch velocity range, no non-periodic regime was manifested in $20 \%$ void fraction. The time history, phase-plane projection, frequency spectrum, and Poincaré map of the tube within $20 \%$ void fraction for some specially chosen flow pitch velocity were shown in Figs. 8 and 9, respectively. As shown in Fig. 8, at $U_{p}=6.998 \mathrm{~m} / \mathrm{s}$, which is larger than the Hopf bifurcation velocity, the trajectory of the tube is toward a stable limit cycle, and the odd frequency in addition to the fundamental frequency can also be observed in Fig. 8(c). One example of the quasi-periodic solution at $U_{p}=9.997 \mathrm{~m} / \mathrm{s}$ is shown in Fig. 9. We note that the frequencies, $\Omega_{1}$ and $\Omega_{2}$, are incommensurate, and the frequency spectrum has peaks at $\Omega_{1}$ $2 \Omega_{2}, \Omega_{1}-\Omega_{2}, \Omega_{1}, \Omega_{1}+\Omega_{2}, \Omega_{1}+2 \Omega_{2}$, et al., as shown in Fig. 9 (c). The points in the Poincaré map of Fig. 9(d) lie on a closed curve, indicating that the corresponding motion is a twoperiodic quasiperiodic.

The bifurcation diagram of the tube in $40 \%$ void fraction was shown in Fig. 10. The Hopf bifurcation in $40 \%$ void fraction occurs at $U_{p}=7.290 \mathrm{~m} / \mathrm{s}$, which is equal to the results obtained from the eigenvalue analysis. When the flow pitch velocity is larger than the threshold of fluidelastic instability, two significant regimes are manifested, the periodic motion regime and the quasi-periodic motion regime. Fig. 11 displays the time history, phase-plane projection, and frequency spectrum of the tube as an example of the periodic motion at $\mathrm{U}_{\mathrm{p}}=11.919 \mathrm{~m} / \mathrm{s}$. And, Fig. 12 displays the time history, phase-plane projection, frequency spectrum, and Poincaré map of the tube as another example of the quasi-periodic motion at $U_{\mathrm{p}}=13.688 \mathrm{~m} / \mathrm{s}$.

Fig. 13 shows the bifurcation diagram for the displacement amplitude of the tube in $60 \%$ void fraction versus the flow pitch velocity. It can be clearly seen that the Hopf bifurcation occurs at $U_{p}=8.280 \mathrm{~m} / \mathrm{s}$. For $U_{p}<14.995 \mathrm{~m} / \mathrm{s}$, the limit cycle motion of the tube can be manifested. One example of the limit cycle motion at $U p=12.688 \mathrm{~m} / \mathrm{s}$ was shown in Fig. 14. When the flow pitch velocity is larger than $14.995 \mathrm{~m} / \mathrm{s}$, the complex periodic motion occurs, as shown in Fig. 15. The complex periodic state cannot be characterized in terms of single frequency as simple periodic oscillation can be. The finite number of points in the Poincaré map shown in Fig. 15(d) confirm the periodicity of the state, which corresponds to a phase-locked motion of the tube.

The bifurcation diagram of the tube in $80 \%$ void fraction was shown in Fig. 16. The Hopf bifurcation occurs at $\mathrm{U}_{\mathrm{p}}=10.080 \mathrm{~m} / \mathrm{s}$. Over a large range of flow pitch velocity above $17.994 \mathrm{~m} / \mathrm{s}$, the periodic motion of the tube can be observed. When the flow pitch velocity is larger than $17.994 \mathrm{~m} / \mathrm{s}$, quasi-periodic motion of the tube occurs. The time history, phase-plane projection, and frequency spectrum of the limit cycle motion for $U_{p}=14.918 \mathrm{~m} / \mathrm{s}$ were shown in Fig. 17, respectively. The time history, phase-plane projection, frequency spectrum, and Poincaré map of the quasi-periodic motion for $U_{p}=18.533 \mathrm{~m} / \mathrm{s}$ were shown in Fig. 18, respectively. A phase-locked motion of the tube can also be observed, as shown in Fig. 18(d).

Summarizing the numerical results obtained, in the singlephase cross-flow ( $0 \%$ void fraction), the tube vibration response may be thought of as comprised of four regimes, the stable regime, the periodic regime, the quasi-periodic regime, and the non-periodic regime. In the stable regime, when the flow pitch velocity is less than the threshold of fluidelastic instability, for any initial conditions, the tube oscillation may decay to zero at

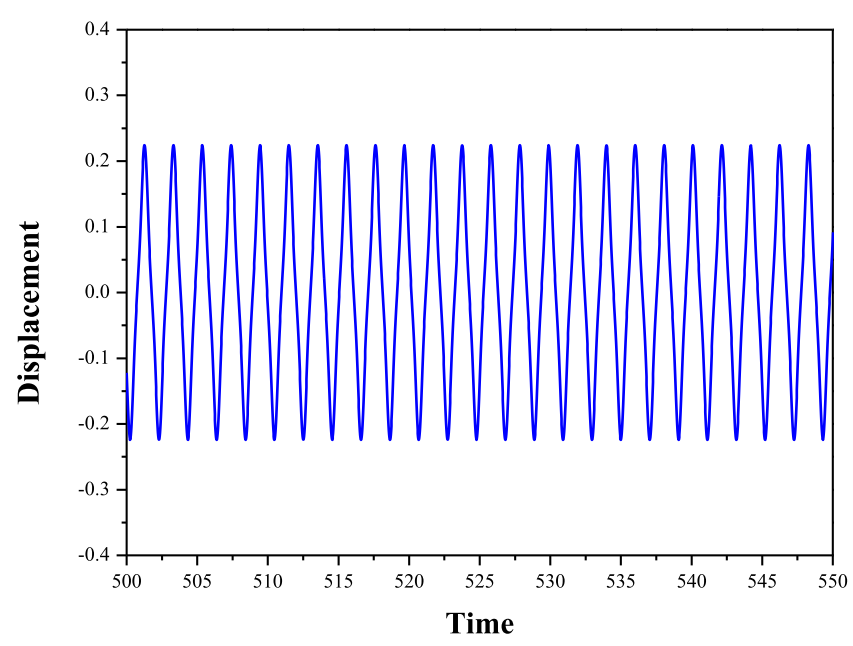

(a)

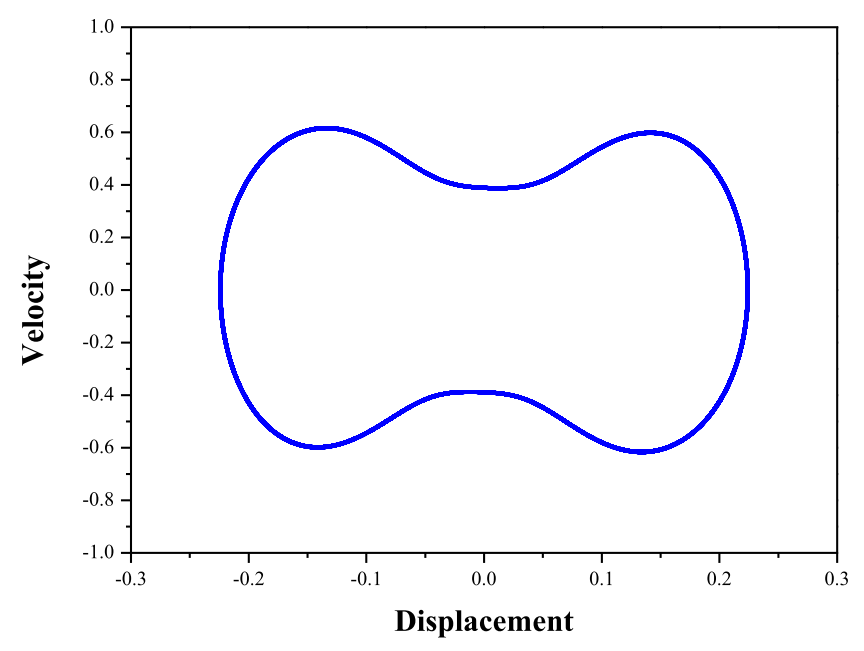

(b)

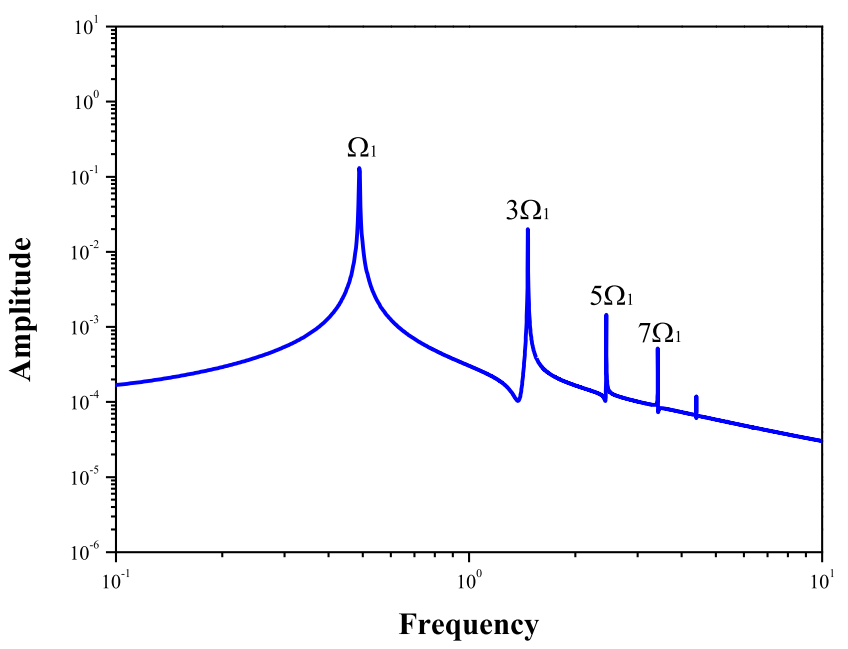

(c)

Fig. 14. Time history, phase-plane projection and spectrum of the tube in $60 \%$ void fraction when the flow pitch velocity is $12.688 \mathrm{~m} / \mathrm{s}$ : (a) displacement; (b) phase plane projection; (c) frequency spectrum. 


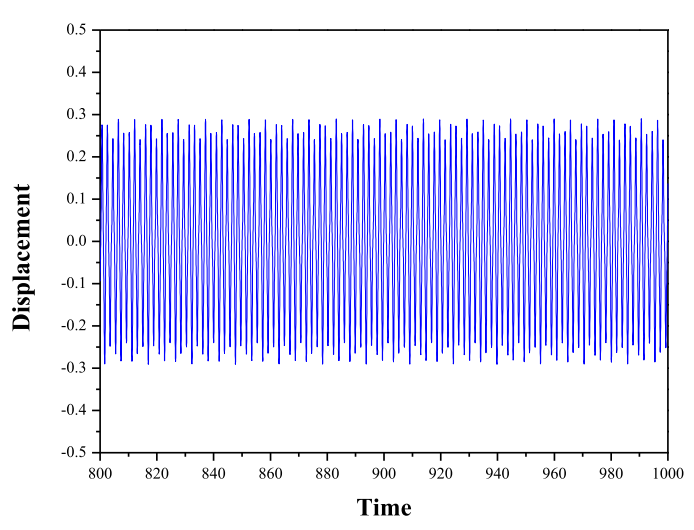

(a)

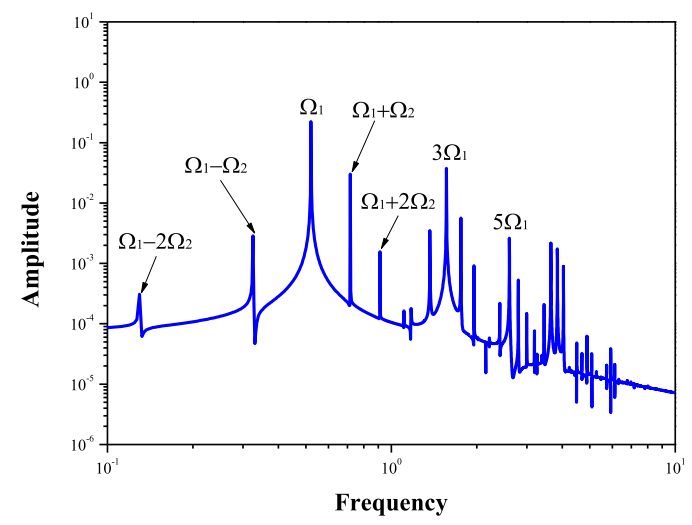

(c)

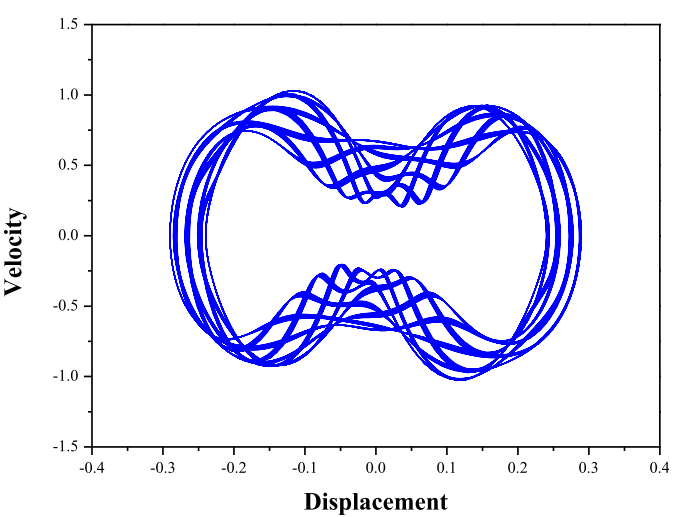

(b)

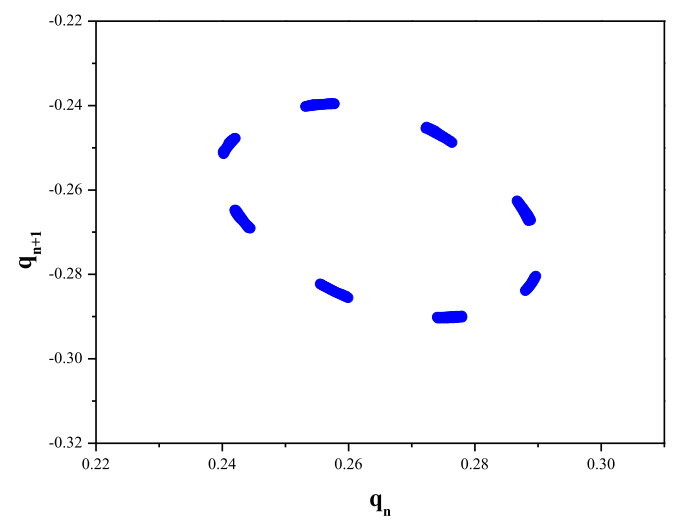

(d)

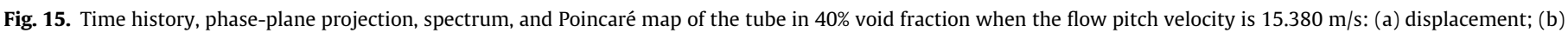
phase plane projection; (c) frequency spectrum; (d) Poincaré map.

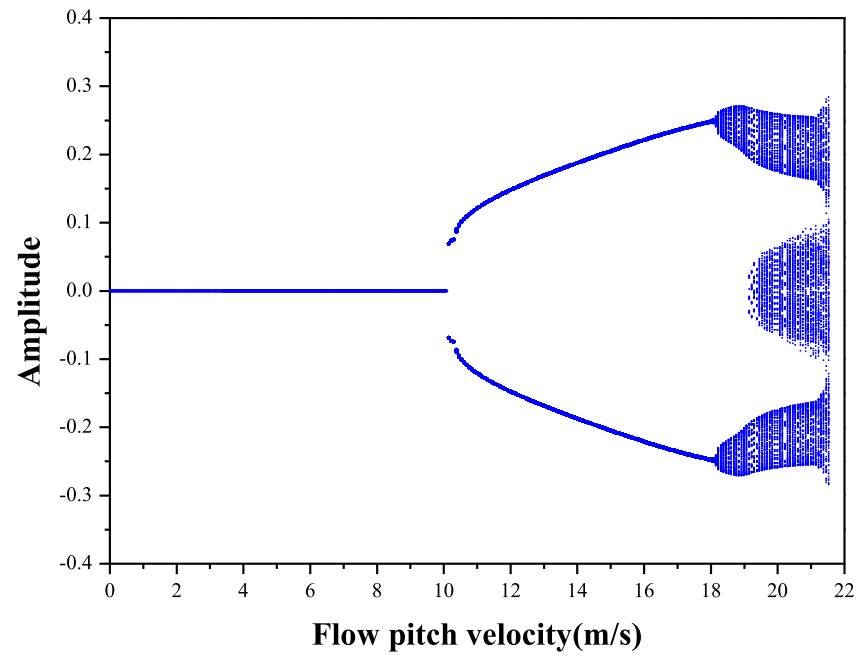

Fig. 16. The bifurcation diagram for the tube bundles with loose support in $80 \%$ void fraction. the equilibrium position. No impact between the tube and tube support plates occurs in this regime. In the periodic regime, Hopf bifurcation occurs, the tube bundles system is unstable. And the amplitude of the limit cycle motion increases monotonously with the flow pitch velocity. The tube collides with the tube support plate at a certain frequency. In the quasi-periodic regime, a quasiperiodic solution is a dynamic solution characterized by two incommensurate frequencies, $\Omega_{1}$ and $\Omega_{2}$. The vibration of the tube is complex. In the non-periodic regime, the influences of the tube support plate are obvious, and the tube vibration becomes much more complicated. The early damage of the tube bundles in a steam generator may be due to the violent collision between the tube and tube support plate.

In addition, within the void fraction of two-phase cross flow ranging from $20 \%$ to $80 \%$, the bifurcation diagrams of the tube are different from the one in single-phase cross-flow. The tube dynamic response in two-phase cross-flow may be thought of as comprised of three regimes, the stable regime, the periodic regime, and the quasi-periodic regime. No non-periodic motion has been observed in this study. It is interesting to note that 


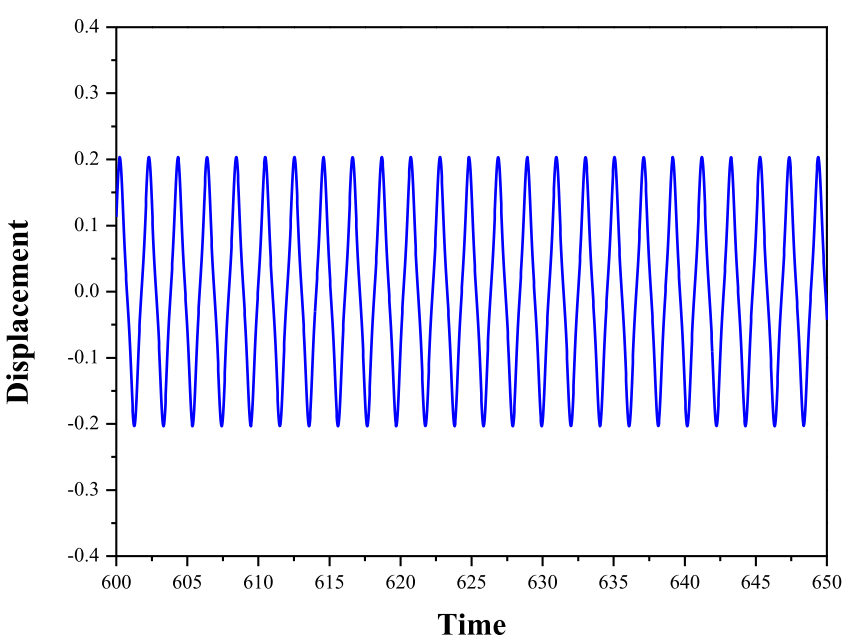

(a)

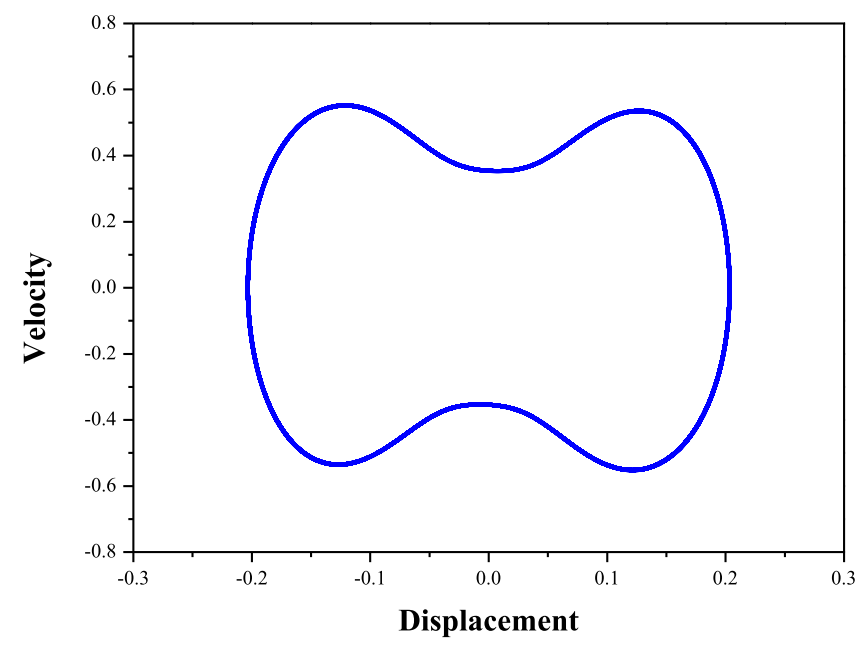

(b)

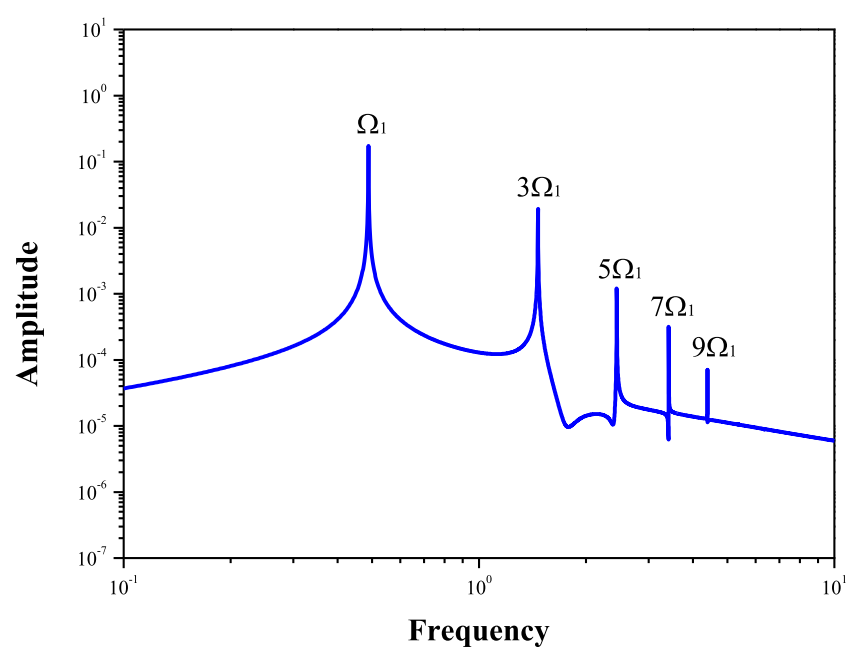

(c)

Fig. 17. Time history, phase-plane projection and spectrum of the tube in $80 \%$ void fraction when the flow pitch velocity is $14.918 \mathrm{~m} / \mathrm{s}$ : (a) displacement; (b) phase plane projection; (c) frequency spectrum. the Hopf bifurcation velocity of the tube increases monotonously as the void fraction of two-phase cross-flow increases. Although the bifurcation diagrams in the two-phase cross-flow are similar, there still exist some differences between the four void fraction conditions considered in this study. In the low void fraction condition (20\% and $40 \%$ void fractions), the typical quasi-periodic motions have been manifested for the high flow pitch velocity. While in the high void fraction (60\% and $80 \%$ void fractions), a phase-locked oscillation has been observed. For examples considered in this study, the Hopf bifurcation velocity in $20 \%, 40 \%, 60 \%$, and $80 \%$ void fraction is $4.940 \mathrm{~m} / \mathrm{s}, 7.270 \mathrm{~m} / \mathrm{s}, 8.290 \mathrm{~m} / \mathrm{s}$, $10.030 \mathrm{~m} / \mathrm{s}$, which is $38.4 \%, 103.6 \%, 132.2 \%$, and $180.9 \%$ more than the Hopf bifurcation velocity in single-phase cross-flow. The velocity of quasi-periodic motion occurs is $8.843 \mathrm{~m} / \mathrm{s}, 13.227 \mathrm{~m} /$ $\mathrm{s}, 15.072 \mathrm{~m} / \mathrm{s}$, and $18.071 \mathrm{~m} / \mathrm{s}$, which is $41.9 \%, 112.3 \%, 141.9 \%$, and $190.1 \%$ more than the quasi-periodic motion occurs in single-phase cross-flow.

\section{Conclusion}

In this paper, a mathematical model of a single flexible simply-supported tube in a rigid tube array subjected to two-phase cross-flow and loose support modeled by a cubic spring has been developed. Based on this model, the eigenvalue problem has been studied firstly. Considering the effect of the loose support, the Hopf bifurcation and various postHopf nonlinear dynamics of the single flexible tube in rigid tube bundles within $0 \%, 20 \%, 40 \%, 60 \%$, and $80 \%$ void fraction were investigated. From these analyses, we have drawn the following conclusions:

1) A supercritical Hopf bifurcation of a single flexible tube in a rotated triangular tube array at the original stable equilibrium occurs at the Hopf bifurcation velocity within void fraction ranging from $0 \%$ to $80 \%$.

2) With increasing the flow pitch velocity of twophase cross-flow beyond the threshold of fluidelastic instability, the amplitude of the limit cycle motion grows monotonously.

3) For sufficiently high flow pitch velocity, the quasi-periodic motion of the tube may occur for both the single-phase cross-flow and two-phase cross-flow conditions. Nonetheless, a significant non-periodic motion has been manifested in the single-phase cross-flow, while the non-periodic motion has not been observed in the two-phase cross-flow conditions.

4) Although the bifurcation diagrams of the tube in two-phase cross-flow for the four void fraction conditions are similar, the vibrations of the single flexible tube in a tube array for the high void fraction condition are much more complex than those for the low void fraction which could be the reason for the early damage of the tube bundles in a steam generator.

\section{CRediT authorship contribution statement}

Jiang Lai: Data curation, Writing - original draft, Software, Formal analysis, Writing - review \& editing. Han Wu: Visualization, Investigation, Formal analysis, Writing - review \& editing. Lei Sun: Conceptualization, Supervision. Lixia Gao: Methodology, Funding acquisition. Pengzhou Li: Supervision. 


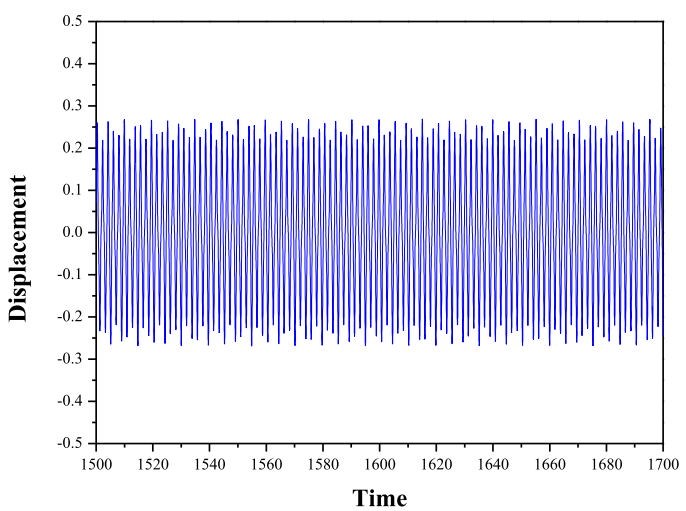

(a)

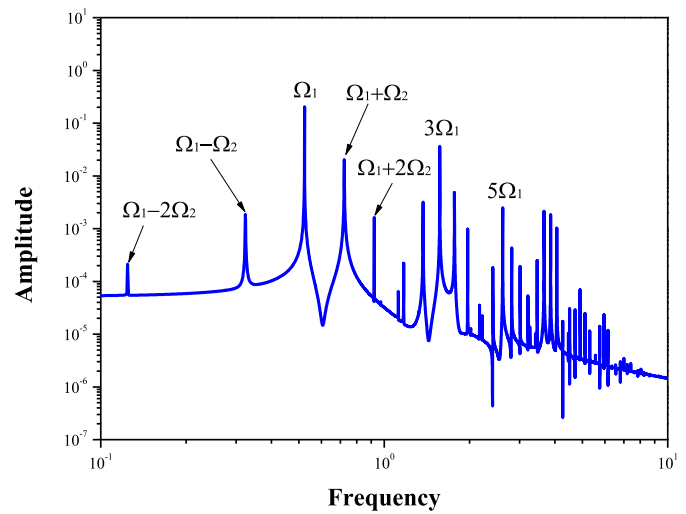

(c)

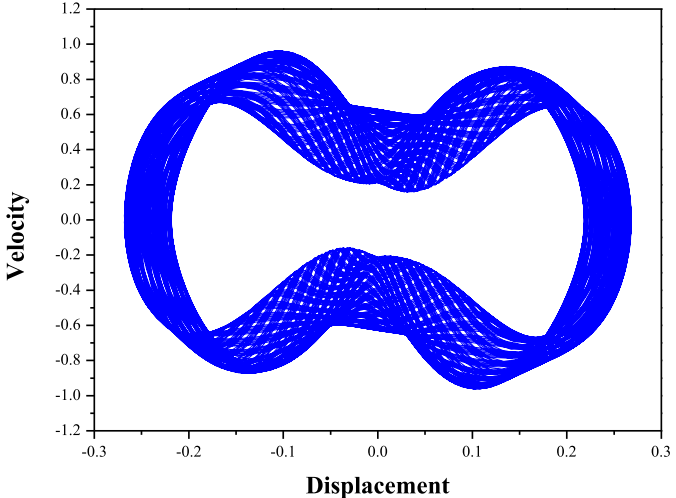

(b)

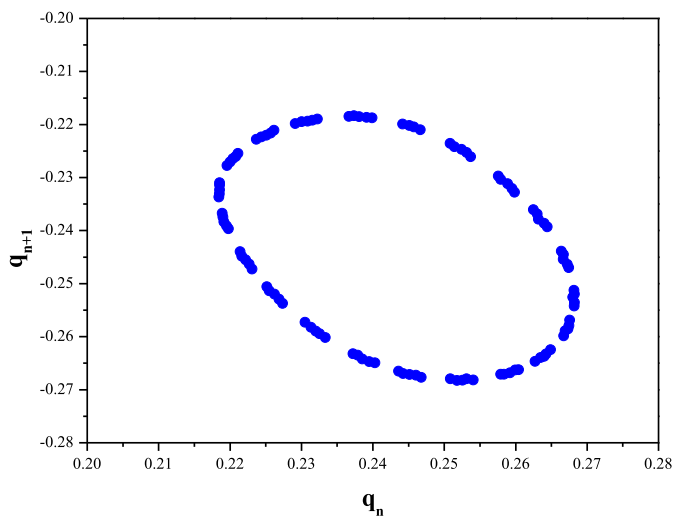

(d)

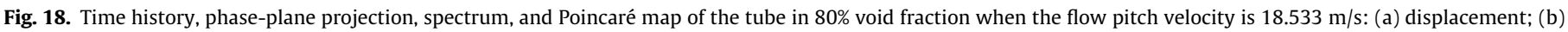
phase plane projection; (c) spectrum; (d) Poincaré map.

\section{Declaration of Competing Interest}

The authors declare that they have no known competing financial interests or personal relationships that could have appeared to influence the work reported in this paper.

\section{Acknowledgments}

The study was made possible by funding from China National Nuclear Corporation and Science Foundation Research Program of Nuclear Power Institute of China.

\section{References}

Abdelbaki, A.R., Paidoussis, M.P., Misra, A.K., 2018. A nonlinear model for a free-clamped cylinder subjected to confined axial flow. J. Fluids Struct. 80, 390404.

Chen, S.S., Cai, Y., Srikantiah, G.S., 1998. Fluid damping controlled instability of tubes in crossflow. J. Sound Vib. 217 (5), 883-907.

Chu, I.C., Chung, H.J., Lee, C.H., 2009. Fluid-elastic instability of rotated square array U-tubes in air-water flow. J. Pressure Vessel Technol. 131. 041301$1 \sim 041301-8$.

Chung, H.J., Chu, I.C., 2006. Fluid-elastic instability of rotated square tube array in an air-water two-phase cross-flow. Nucl. Eng. Technol. 38 (1), 69-80.

Bouzidi, S.El., Hassan, M., 2015. An investigation of time lag causing fluidelastic instability in tube arrays. J. Fluids Struct, 57, 264-276.

Jiang, N., Bin, C., Zang, F., Zhang, Y., 2015. An unsteady model for fluidelastic instability in an array of flexible tubes in two-phase cross-flow. Nucl. Eng. Des. 285, 58-64.

Jiang, N., Xiong, F., Zang, F., Zhang, Y., Qi, H., 2017. Analysis on vibration response of U-tube bundles caused by two-phase cross-flow turbulence. Ann. Nucl. Energy 99, 328-334.
Joo, Y., Dhir, V.K., 1995. On the mechanism of fluidelastic instability of a tube placed in an array subjected to two-phase crossflow. J. Fluids Eng. 117, 707-712.

Lai, J., Sun, L. Li, P. Tan, T. Gao, L. Xi, Z. He, C., 2019. Eigenvalue analysis on fluidelastic instability of a rotated triangular tube array considering the effects of two-phase flow. J. Sound Vib. 439, 194-207.

Lai, J., Sun, L., Li, P., 2019. Flow-induced instability and nonlinear dynamics of a tube array considering the effect of a clearance gap. Nucl. Eng. Technol. 51 (6), 16731680.

L. Liu J. Feng H. Wu, W.X, W. Tan. Fluid excitation forces on a tightly packed tube bundle subjected in cross-flow. J. Press. Vess. Technol. 1392017 0313071 031307-8.

Liu, H., Lai, J., Sun, L., Li, P., Gao, L., Yu, D., 2019. Fluidelastic instability of a tube array in two-phase cross-flow considering the effect of tube material. Nucl. Eng. Technol. 51, 2026-2033.

Ma, Y., Luan, Y., Xu, W., 2020. Hydrodynamic features of three equally spaced, long flexible cylinders undergoing flow-induced vibration. Eur. J. Mechan.-B/Fluids 79, 386-400.

Mahon, J., Meskell, C., 2009. Investigation of the underlying cause of the interaction between acoustic resonance and fluidelastic instability in normal triangular tube arrays. J. Sound Vib. 324, 91-106.

Ni, Q., Wang, Y., Tang, M., Luo, Y., Yan, H., Wang, L., 2015. Nonlinear impacting oscillations of a fluid-conveying pipe subjected to distributed motion constraints. Nonlinear Dyn. 81, 893-906.

Paidoussis, M.P., Li, G.X., 1992. Cross-flow-induced chaotic vibrations of heat-exchanger tubes impacting on loose supports. J. Sound Vib. 152 (2), 302-326.

Paidoussis, M.P., Li, G.X., Moon, F.C., 1989. Chaotic oscillations of the autonomous system of a constrained pipe conveying fluid. J. Sound Vib. 135, 1-19.

Paidoussis, M.P., Li, G.X., Rand, R.H., 1991. Chaotic motions of a constrained pipe conveying fluid: comparison between simulation, analysis and experiment. J. Appl. Mech. 58, 559-565.

Piteau, P., Delaune, X., Antunes, J., Borsoi, L., 2012. Experiments and computations of a loosely supported tube in a rigid bundle subjected to single-phase flow. J. Fluids Struct. 28, 56-71.

Ricciardi, G., Pettigrew, M.J., Mureithi, N.W., 2011. Fluidelastic instability in a normal triangular tube bundle subjected to air-water cross-flow. J. Pressure Vessel Technol. 133. 061301-1 061301-9. 
Sadek, O., Mohany, A., Hassan, M., 2018. Numerical investigation of the cross flow fluidelastic forces of two-phase flow in tube bundle. J. Fluids Struct. 79, 171186.

Shinde, V., Longatte, E., Baj, F., Braza, M., 2018. A theorical model of fluidelastic instability in tube arrays. Nucl. Eng. Des. 337, 406-418.

Wang, L., Dai, H.L., Han, Y.Y., 2012. Cross-flow-induced instability and nonlinear dynamics of cylinder arrays with consideration of initial axial load. Nonlinear Dyn. 67, 1043-1051.
Zhang, X., Jiang, B., Zhang, L., Xiao, X., 2016. Fluid-elastic instability tests on paralle triangular tube bundles with different mass ratio values under increasing and decreasing flow velocities. Shock Vib. No, 1680218.

Zhao, W., Xue, F., Shu, G., Liu, M., Lin, L., Wang, Z., 2014. Analysis of flow-induced vibration of steam generator tubes subjected to cross flow. Nucl. Eng. Des. 275, 375-381. 